\title{
Un Estudio de Validación de una Escala de Evaluación del Constructo de Justicia Social Percibida en Educación Primaria y Secundaria
}

\author{
Validation Study of an Evaluation Scale of 'Perceived Social \\ Justice' in the Primary and Secondary Education
}

\author{
Anna Jordina Llorens * \\ Jesús Miguel Jornet \\ Purificación Sánchez-Delgado \\ Universitat de València, España
}

\begin{abstract}
La evaluación educativa se ha basado fundamentalmente en el éxito académico. Sin embargo, estudios recientes han valorado estudiar la percepción que el propio alumnado tiene sobre otros aspectos como, por ejemplo, el de la justicia social. Así pues, el objetivo general de nuestro trabajo es el diseño de un instrumento que permita evaluar la percepción que crea el alumnado sobre justicia social educativa (JSP-E) a partir de las interacciones y relaciones que se establecen entre alumnado y profesorado. El constructo "Justicia Social Percibida en la Educación" se incluye dentro de la dimensión de equidad del modelo de evaluación de instituciones y sistemas educativos definido en Jornet (2012) dirigido a valorar la aportación de la educación para el desarrollo personal y la transformación social. Para ello, los jueces llevarán a cabo diferentes fases de valoración (relevancia, adecuación, susceptibilidad de cambio y claridad), en donde se considerarán cada uno de los criterios para recoger dicha percepción. Finalmente, también, se reflejan en este artículo las puntuaciones de valoración de los jueces y la propuesta final de la escala que queremos trabajar.
\end{abstract}

Descriptores: Justicia social; Docente; Percepción; Evaluación; Método de evaluación.

\begin{abstract}
The educational evaluation has been based fundamentally on academic success. However, recent studies have valued studying the perception that the students themselves have about other aspects such as, for example, that of social justice. Therefore, the general objective of our work is the design of an instrument that allows evaluating the perception that students create about educational social justice (JSP-E) from the interactions and relationships established between students and teachers. The construct "Social Justice Perceived in Education" is included within the equity dimension of the model of evaluation of institutions and educational systems defined in Jornet (2012) aimed at assessing the contribution of education for personal development and social transformation. To do this, the judges will carry out different phases of assessment (relevance, adequacy, susceptibility to change and clarity), where each of the criteria for collecting said perception will be considered, and finally, this article will also reflect the evaluation of the judges and the final proposal of the scale that we want to work.
\end{abstract}

Keywords: Social justice; Teachers; Perceptions; Evaluation; Evaluation methods.

*Contacto: anjorllo@alumni.uv.es

ISSN: 2254-3139

www.rinace.net/riejs/

revistas.uam.es/riejs
Recibido: $\quad 19$ de septiembre 2017

$1^{\text {a }}$ Evaluación: 9 de diciembre 2017

$2^{\text {a }}$ Evaluación: 23 de abril 2017

Aceptado: $\quad 3$ de mayo 2018 


\section{Introducción}

En trabajos anteriores (Jornet, Sancho y Bakieva, 2015a; Llorens, 2016) avanzamos en la definición de un constructo relativo a la percepción de justicia social que el alumnado va construyendo a partir de sus vivencias escolares. El constructo "Justicia Social Percibida en la Educación (JSP-E, en lo sucesivo)" se incluye dentro de la dimensión de equidad del modelo de evaluación de instituciones y sistemas educativos definido en Jornet (2012) dirigido a valorar la aportación de la educación para el desarrollo personal y la transformación social. Desde esta misma concepción de equidad, es como nosotros concebimos la evaluación como justa (Hidalgo, 2017), independientemente del método de recogida de información que se establezca.

Ya en Llorens (2016) comenzamos a diseñar las bases del instrumento JSP-E. En este caso, nuestro interés se orienta a medir la percepción individual de justicia de los alumnos, respecto a la interacción y las relaciones que establecen con otros agentes educativos. A partir de Llorens (2016), pudimos perfilar la definición inicial de Jornet, Sancho y Bakieva (2015a) incidiendo en el valor del efecto de las interrelaciones que se dan entre los agentes involucrados en la escuela.

Como constructo, entendemos que la JSP-E se configura precisamente a partir de las vivencias que tiene el alumnado en la escuela, tanto con sus iguales, como en relación con el profesorado. Por lo tanto, se trata de una construcción personal que, naciendo desde las emociones y las percepciones, se va convirtiendo en sentimiento arraigado y se va constituyendo en una forma de asumir su relación con el mundo, orientando sus actos, previendo sus consecuencias y valorando si las mismas son contingentes con las expectativas que cada cual se crea. Es importante considerarlo así, porque -como hipótesis interpretativa que orienta esta propuesta- entendemos que se relacionará con el esfuerzo, con la implicación que el alumnado pueda estar dispuesto a ofrecer, con el Valor Social Subjetivo que se le da a la educación (VSE-S; Sancho, Jornet y González-Such, 2016), con la resiliencia y con la motivación. Se trata pues de un constructo socio-afectivo, de carácter evolutivo y que puede llegar a condicionar la acción del alumnado, creando una visión facilitadora u obstaculizadora. Con independencia de las consecuencias inmediatas para la vida escolar, es posible que contribuya a la visión global que, como persona, se configura cada cual acerca de la justicia social en mayúsculas; es decir, aquélla que nos encontraremos o no, a lo largo de toda la vida. Obviamente, ello es un aspecto que va más allá de cualquier pretensión de investigación a corto plazo, puesto que su estudio requeriría de un seguimiento longitudinal que no podemos prever si sería posible realizar en algún estudio a medio-corto plazo como éste. Por ello, nos centramos en lo inmediato, en aquello que es aprehensible en el escenario escolar.

Así pues, este modelo hace referencia a cómo nos afecta el contexto en que vivimos y cómo sus cambios pueden crearnos expectativas personales que nos influyen en nuestra manera de pensar y de concebir el mundo que nos rodea. Tal y como hemos señalado, pensamos que las vivencias de justicia/injusticia social que se dan en el contexto educativo van permeando en el alumnado a través de sus emociones y van construyendo el sentimiento de lo que consideran como existencia o inexistencia de justicia social.

Por otra parte, es conveniente insistir en los puntos de vista que compartimos con diversos autores (Connell, 1997; Hidalgo, 2017; Jornet, 2012; Lundgren, 2013) acerca de las reflexiones básicas para evaluar los sistemas educativos, además de las competencias 
cognitivas que todos aquellos constructos psico-socio-afectivos deben tener para promover la finalidad principal de la educación: el desarrollo integral de la persona ${ }^{1}$. Es por ello que fomentar valores más allá de las materias académicas ${ }^{2}$ es uno de nuestros intereses principales a la hora de promover evaluaciones de sistemas educativos justos. En definitiva, la tarea educativa debe trabajar para desarrollar mejores personas para que, a su vez, puedan mejorar la sociedad. Otros autores dirían que nuestra perspectiva trata de fomentar una educación orientada hacia una concepción de Sostenibilidad Social. Llámese como se considere, promover evaluaciones justas es nuestra preocupación principal y por ello queremos recoger las percepciones del alumnado para que, a partir de ahí, podamos iniciar una propuesta de evaluación diferente a la actual, una evaluación que sin duda nosotros consideramos como justa y en la que el alumnado sea el verdadero protagonista no sólo como fin, sino como individuo que necesita ser comprendido, escuchado y valorado. Porque si de verdad creemos que los educandos son los protagonistas de la educación, ¿ipor qué la mayoría de las veces sus percepciones quedan ninguneadas a la hora de aplicar cambios educativos? ¿Hasta qué punto quedan reflejados sus intereses en los informes de calado internacional?

Así pues, el objetivo general del trabajo que aquí presentamos es el diseño de un instrumento que permita evaluar la percepción que tiene el alumnado de la Justicia Social Percibida en Educación (referida a la escuela o a la justicia escolar; JSP-E), a partir de las interacciones y relaciones que se establecen entre alumnado y profesorado. Los objetivos específicos son:

- Realizar una aproximación al estado del arte acerca del desarrollo teórico, investigador y evaluativo de la JSP-E por el alumnado.

- Establecer una definición operativa del constructo, y su traducción en dimensiones, indicadores e ítems de evaluación.

- Aportar una primera evidencia de validación de constructo (y de contenido) a partir del análisis de expertos o jueces.

- Diseñar una escala que permita su posterior análisis empírico en cuanto a sus propiedades métricas y evidencias de validación.

- Establecer una propuesta final que pueda ser aplicada en un estudio piloto.

\section{Revisión de la literatura}

Desde la propuesta de Connell (1997), pasando por las propuestas más actuales de Murillo e Hidalgo (2015), hemos considerado oportuno tener en cuenta la definición del término de JSP-E validada por Jornet, Sancho y Bakieva (2015a, p. 118)3 y en base a ella matizamos su operativización en Llorens (2016, p. 42):

\footnotetext{
${ }^{1}$ Véase que las opiniones y las percepciones del alumnado ya se tenían en cuenta para el ámbito de la evaluación educativa dentro de la taxonomía de objetivos educativos de Bloom y otros (1956).

2 Téngase en cuenta, por ejemplo, las fundamentaciones que promulga la ética del cuidado. Respecto a ella, véanse las aportaciones de autores como Neufeld (1992); Fernández Herrería y López López (2010); Murga-Menoyo y Novo Villaverde (2014), entre otros.

${ }^{3}$ Téngase en cuenta que esta definición se realiza dentro del modelo de evaluación para la Cohesión Social (Jornet 2012 ) por lo que los límites de la definición de este constructo se establecen en relación a las definiciones de los demás constructos, por lo que pueden haber variables que se asocien a estos otros constructos también.
} 
La imagen que se construye de ésta (JSP-E) a partir de las relaciones y las interacciones que se crean en el ámbito escolar con todos sus integrantes: el equipo directivo, el profesorado, el alumnado y otras personas involucradas en el contexto académico.

Como características intrínsecas del concepto aquí definido, nótese que se enfatiza de la siguiente manera:

- Se trata de una percepción del alumnado, por lo que los mismos acontecimientos o situaciones no necesariamente tienen que ser interpretados de la misma manera por todo el alumnado, sino que cada cual filtra la realidad según sus construcciones personales, a la par que los nuevos acontecimientos pueden ir reconfigurando tales construcciones ${ }^{4}$.

- Se construye a partir de las relaciones e interacciones personales/sociales que se dan en el entorno escolar.

- No se circunscribe a lo que se produce respecto al alumno particular que responde como protagonista de la acción, sino que incluye todo lo que observa y le impacta en su forma de ver e interpretar lo que sucede en el mundo escolar en el que se desarrolla.

La preocupación por el desarrollo de la justicia social en la escuela implica el desarrollo global de la persona considerando, además de los conocimientos cognitivos necesarios, los valores cívico-éticos, democráticos y sociales a adquirir (Connell, 1997). Como constructo socio-afectivo se desarrollará construyendo el entramado de construcciones personales que definirán la forma de ser y estar de la persona en el mundo; es decir, de una u otra manera formará parte del proceso de configuración de su personalidad.

Primeramente, para poder iniciar nuestro estudio de JSP-E hemos realizado una revisión pormenorizada de aquellos trabajos que presentan un instrumento que pretende analizar de alguna manera la justicia social percibida en ámbitos educativos. Es por ello que en el cuadro 1 hemos querido recoger una síntesis de aquellos estudios que consideramos relevantes y que están estrechamente vinculados con la propuesta de JSP-E. De entre los revisados asumimos como puntos de partida para la operativización del constructo y diseño del instrumento los trabajos de Ludlow, Enterline y Cochran-Smith (2008) y Jacott y otros (2014) (cuadro 1).

En concreto, para comenzar con el diseño de nuestro instrumento, se ha procedido a seleccionar la información teórica más relevante a partir del trabajo de Connell (1997), aunque éste no presentara ningún instrumento, hasta pasar por aquellos más actuales que sí presentan un instrumento.

Respecto a las bases teóricas del constructo, principalmente se han tenido en cuenta las reflexiones de Jornet, Sancho y Bakieva (2015b) y de Fraser (2008) para valorar la justicia educativa como "reconocimiento", ya que sus propuestas también se han identificado en otros trabajos posteriores con preocupaciones similares (Hidalgo, 2017).

\footnotetext{
${ }^{4}$ En definitiva, nos basamos en la Teoría de los Constructos Personales (TCP), formulada por Kelly (1955) y que dio origen al denominado alternativismo constructivista en las teorías de la personalidad, descrito por Rivas, Alcantud y Gaya (1994).
} 
Cuadro 1. Selección de investigaciones que consideran la Justicia Social dentro del ámbito educativo

\begin{tabular}{|c|c|c|}
\hline INSTRUMENTOS & INTERÉS DEL INSTRUMENTO & $\begin{array}{c}\text { AUTOR/ES Y } \\
\text { FECHA }\end{array}$ \\
\hline $\begin{array}{l}\text { Escala de creencias: } \\
\text { Aprendiendo a Enseñar } \\
\text { para la Justicia Social. }\end{array}$ & $\begin{array}{l}\text { Se evalúan las actitudes docentes hacia el } \\
\text { aprendizaje de la Justicia Social en el } \\
\text { ámbito educativo. }\end{array}$ & $\begin{array}{l}\text { Ludlow, Enterline } \\
\text { y Cochran-Smith } \\
(2008) \text {. }\end{array}$ \\
\hline $\begin{array}{l}\text { Escala de Justicia Social } \\
\text { para los futuros maestros. }\end{array}$ & $\begin{array}{l}\text { Se analizan los principios de equidad, } \\
\text { sensibilidad social, responsabilidad social } \\
\text { y percepción de situaciones de } \\
\text { discriminación. }\end{array}$ & Turhan (2010). \\
\hline Escala LTSJ-B. & $\begin{array}{l}\text { Actitudes docentes y creencias sobre la } \\
\text { Justicia Social en el ámbito educativo. Con } \\
\text { ella se recogieron datos de } 304 \text { profesores } \\
\text { australianos en formación. }\end{array}$ & $\begin{array}{l}\text { Cochran-Smith y } \\
\text { otros }(2012) \text {. }\end{array}$ \\
\hline Escala LTSJ-B mejorada. & $\begin{array}{l}\text { Actitudes docentes y creencias sobre la } \\
\text { Justicia Social en el ámbito educativo. }\end{array}$ & $\begin{array}{l}\text { Ginns y otros } \\
(2014) \text {. }\end{array}$ \\
\hline $\begin{array}{l}\text { Cuestionario de Justicia } \\
\text { Social. }\end{array}$ & $\begin{array}{l}\text { Evaluación de las concepciones sobre } \\
\text { Justicia Social en el ámbito escolar por } \\
\text { docentes y alumnado de Educación } \\
\text { Primaria y Secundaria. }\end{array}$ & $\begin{array}{l}\text { Jacott y otros } \\
(2014) \text {. }\end{array}$ \\
\hline $\begin{array}{l}\text { Cuestionario de Justicia } \\
\text { Social percibida para } \\
\text { alumnado universitario en } \\
\text { el contexto estadounidense. }\end{array}$ & $\begin{array}{l}\text { Escalas valoradas sobre creencias y } \\
\text { percepciones en: } \\
\text { Justicia en el Mundo } \\
\text { Racismo simbólico } \\
\text { Neosexismo } \\
\text { Motivación por los Servicios Públicos } \\
\text { Los resultados y las implicaciones de } \\
\text { estos análisis son analizados y discutidos } \\
\text { en el mismo artículo. }\end{array}$ & $\begin{array}{l}\text { Torres-Harding y } \\
\text { otros (2014). }\end{array}$ \\
\hline $\begin{array}{l}\text { Modelos evaluativos que } \\
\text { parten de la observación } \\
\text { analítica: Learning Record. }\end{array}$ & $\begin{array}{l}\text { En el estudio se valoran aquellos métodos } \\
\text { evaluativos que perpetúan injusticas sin } \\
\text { tener en cuenta las necesidades y } \\
\text { características particulares del alumnado } \\
\text { durante el proceso de enseñanza- } \\
\text { aprendizaje. }\end{array}$ & $\begin{array}{l}\text { Syverson (2009). } \\
\text { Barr y Tagg } \\
(1998)\end{array}$ \\
\hline
\end{tabular}

Fuente: Elaboración propia a partir de Jornet, Sancho y Bakieva (2015a, p. 119).

Por otra parte también, para poder comprender adecuadamente la definición de este constructo, debemos tener en cuenta cuáles son las dimensiones en las que JSP-E se divide o en qué escenarios se enmarca dentro de la vida escolar. Esto son: a) el centro, como macro-escenario en el que se producen las interacciones más alejadas y difusas, pero en el que se manifiesta un clima de relaciones que puede llegar a ser percibido por el alumnado, y b) el aula, micro-escenario más cercano al alumnado, en donde éstos interaccionan con semejantes y profesorado respecto a tareas y normas específicas. Sin ninguna duda, ambos escenarios constituyen las dimensiones de análisis situacional del constructo de JSP-E.

Además, en el estudio de la JSP-E, no podemos dejar de lado a los profesionales de la educación, sobretodo, a los docentes. Porque son ellos quienes constituyen el modelo de referencia en el proceso educativo formal. Son quienes, mediante sus interacciones, sus mensajes y sus conductas proyectan sobre el alumnado conocimientos, sentimientos y experiencias. Son quienes pueden reconducir las situaciones educativas. Pero, ¿qué pasa cuando las circunstancias impiden que el alumnado logre lo que desee lograr? ¿La docencia otorga las mismas oportunidades de actuación a todo el alumnado? ¿Qué 
facilidades aporta el profesorado o qué ayudas aportan éstos a sus alumnos? ¿Se muestra al alumnado cuáles son sus derechos, obligaciones y libertades? Otra de las preocupaciones que nos concierne, y que coincide con la declaración de Educación para el Desarrollo Sostenible (UNESCO, 2012), es de si se trata a todo el alumnado de manera equitativa, independiente de su origen étnico, género o características particulares. Y lo que es más importante, ¿ise les otorga el mismo respeto a todos? Y otra duda más, ¿todo el alumnado se siente igual de valorado por todos sus profesores? Éstas y otras son las cuestiones centrales a evaluar en un sistema educativo justo (Hidalgo, 2017), y por ello queremos conocer qué percepción tiene el alumnado respecto a estas cuestiones. Además, a la hora de aplicar nuestra propuesta, debemos tener en cuenta que consideramos al profesorado como un modelo esencial de autoridad del que, de su buena o mala acción, dependerá el ejercicio de justicia (asumiblemente distributiva) y que, en consecuencia, el alumnado percibirá de manera positiva o negativa.

\section{Método}

A continuación, indicamos las fases de valoración y los criterios trabajados que se han tenido en cuenta para el diseño del instrumento final (figuras 1 y 2). Respecto a los análisis métricos, este estudio se enmarca en el enfoque de análisis de evidencias de validez desde una concepción unitaria (APA, AERA y NCME, 2014), que se describen en Leyva (2004), Ruiz-Primo, Jornet y Backhoff (2006), Bakieva (2016) y Jornet, González-Such y Bakieva (2017) (figura 3).

\subsection{Participantes}

En las figuras siguientes (cuadros 2 y 3 ) se presenta un desglose pormenorizado de las características personales de los jueces que han participado en las diversas fases de valoración. El único filtro que se ha realizado para escoger a los jueces ha sido la consideración de que en su conjunto debían cubrir todos los ambientes educativos. Para ello, se ha intentado trabajar con un grupo de profesionales que posea una experiencia profesional amplia y variada, en donde los jueces participantes tienen bagaje teóricopráctico de al menos una de las siguientes profesiones: orientador, maestro de infantil y primaria, profesor de secundaria y FP, profesor de universidad, educador social, orientador y otros campos (inspector de educación, logopeda, psicólogo, etc.).

En total son 30 los jueces que han valorado la escala de relevancia y susceptibilidad de cambio y 26 los que han valorado la escala de claridad y adecuación.

En la fase de valoración de relevancia y susceptibilidad de cambio, respecto a los jueces participantes distribuidos por edad, encontramos que el grupo mayoritario corresponde al intervalo de 21 a 30 años (37\%), seguido del intervalo de 31 a 40 años (30\%), del intervalo de 51 a 60 años (20\%) y finalmente del intervalo de 41 a 50 años (13\%). Por tanto, podemos decir que es un grupo de jueces relativamente joven.

En cuanto a la profesión de los jueces participantes en esta fase, el grupo más numeroso corresponde a los profesores de universidad (33\%), seguido del grupo "otros" en el que se incluyen profesiones tales como psicólogos, logopedas e inspectores de educación (27\%), seguido del grupo de maestros de infantil y primaria (13\%), profesores de secundaria y FP (13\%) y el grupo de orientadores y educadores sociales (13\%). 


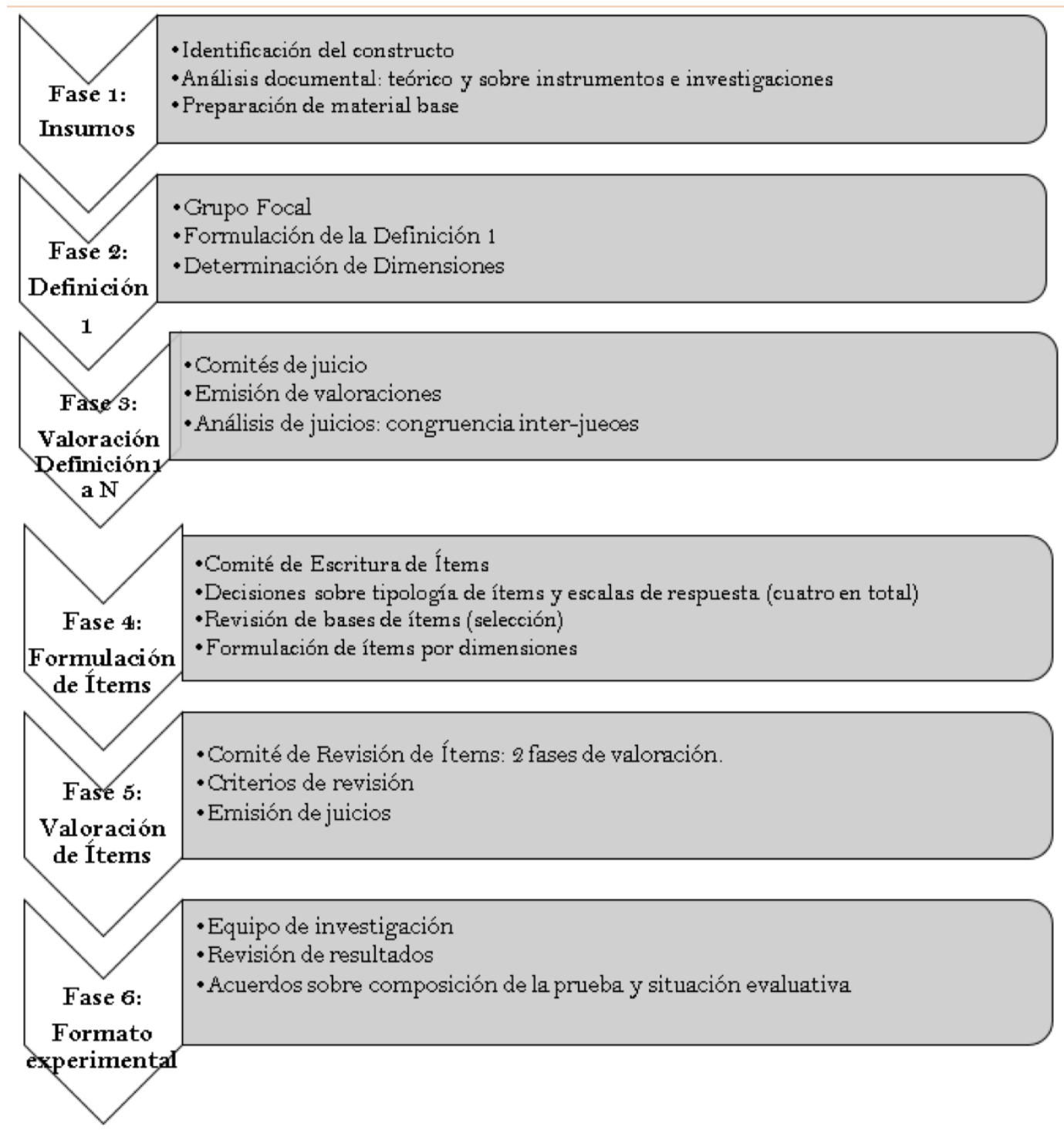

Figura 1. Fases para la definición y operativización de constructos para instrumentos de medida

Fuente: Elaborado a partir de Jornet, González-Such y Bakieva (2017).

En cuanto a los años de experiencia profesional, podemos decir que es un grupo de participantes compuesto sobre todo por profesionales de corta experiencia (56\%), lo que significa que han trabajado hasta 10 años. Así, el 44\% de participantes son profesionales con más de 10 años de experiencia profesional.

En la fase de valoración de claridad y adecuación, los jueces participantes distribuidos por edad, tienen características similares al caso anterior, puesto que el grupo mayoritario corresponde al intervalo de 21 a 30 años (35\%), seguido del intervalo de 31 a 40 años (27\%), del intervalo de 51 a 60 años (23\%) y finalmente del intervalo de 41 a 50 años (15\%). Por tanto, podemos decir que también es un grupo de jueces relativamente joven. 


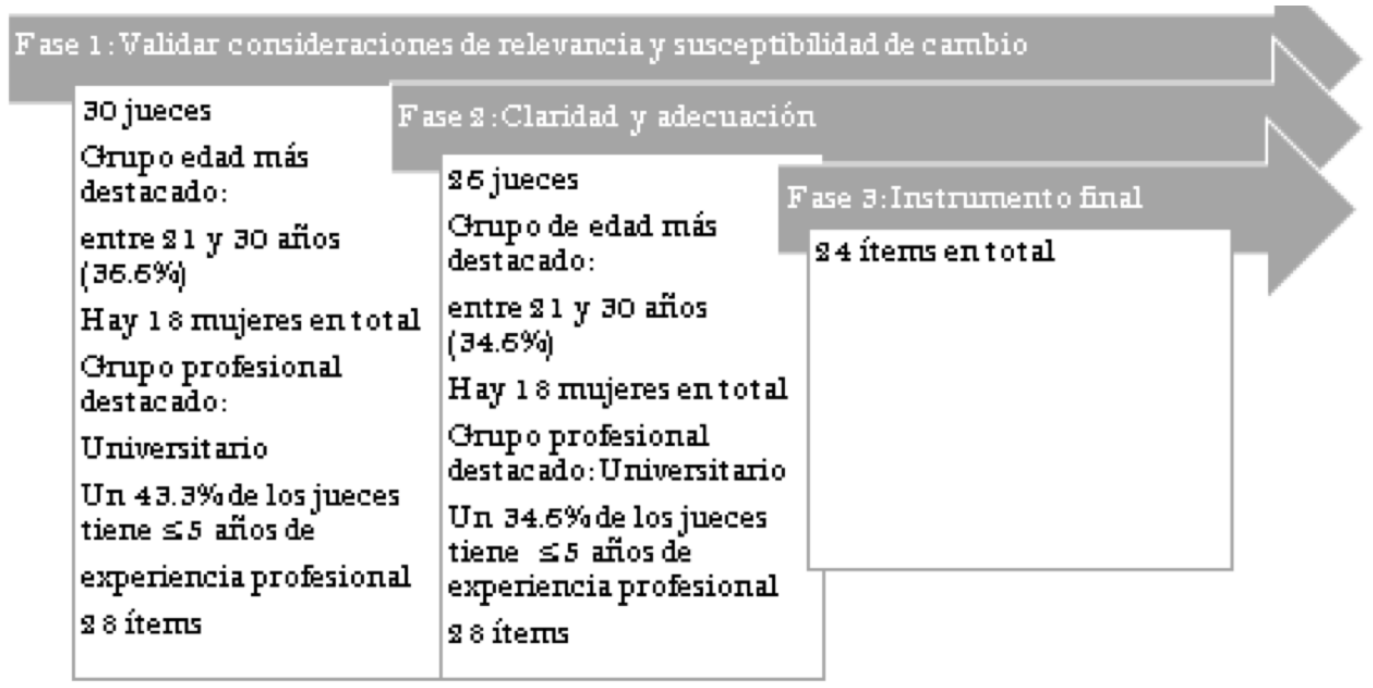

Figura 2. Proceso de valoración y grupos de jueces

Fuente: Elaboración propia.

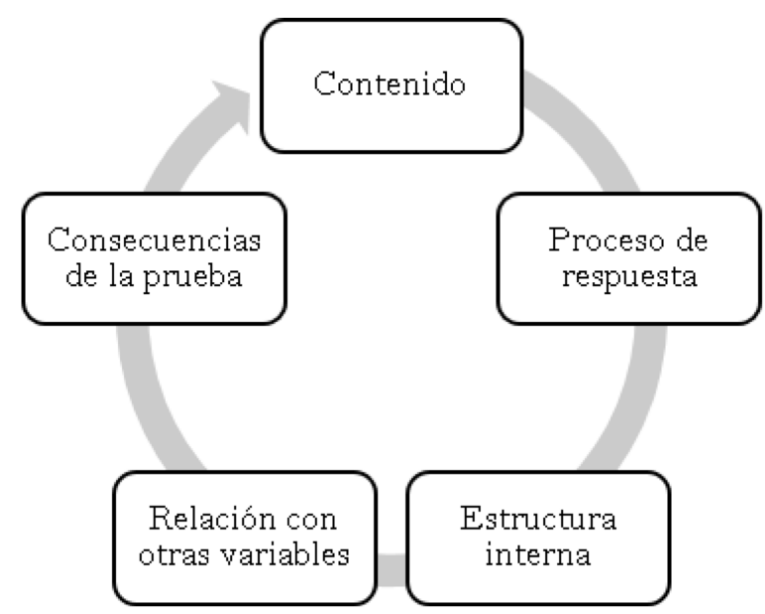

Figura 3. Validez como concepto unitario (APA, AERA, y NCME, 2014)

Fuente: Recuperado de Bakieva (2016, p. 140).

En cuanto a la profesión de los jueces participantes en esta fase, el grupo más numeroso corresponde a los profesores de universidad (38\%), seguido del grupo "otros" en el que se incluyen profesiones tales como psicólogos, logopedas e inspectores de educación (23\%), profesores de secundaria y FP (15\%), maestros de primaria (12\%), y especialistas como orientadores y educadores sociales (12\%).

Por último, en cuanto a los años de experiencia profesional, podemos decir que es un grupo de participantes compuesto tanto por profesionales de corta experiencia $(51 \%)$, como por profesionales de más de 10 años de experiencia (49\%). 
Cuadro 2. Grupo de jueces para valorar escalas de relevancia y susceptibilidad de cambio

\begin{tabular}{|c|c|c|c|c|c|c|c|}
\hline & & & BRE & & IER & & TAL \\
\hline & & N. & $\%$ & N. & $\%$ & N. & $\%$ \\
\hline & $51-60$ & 3 & 10 & 3 & 10 & 6 & 20 \\
\hline & $41-50$ & 1 & 3,3 & 3 & 10 & 4 & 13,3 \\
\hline Edad & $31-40$ & 4 & 13,3 & 5 & 16,6 & 9 & 30 \\
\hline & $21-30$ & 4 & 13,3 & 7 & 23,3 & 11 & 36,6 \\
\hline & Total & 12 & 40 & 18 & 60 & 30 & 100 \\
\hline & Orientador & 1 & 3,3 & 1 & 3,3 & 2 & 6,6 \\
\hline & Infantil & & & 1 & 3,3 & 1 & 3,3 \\
\hline & Primaria & & & 3 & 10 & 3 & 10 \\
\hline & Secundaria & 2 & 6,6 & 1 & 3,3 & 3 & 10 \\
\hline Profesión & $\mathrm{FP}$ & 1 & 3,3 & & & 1 & 3,3 \\
\hline & Universidad & 5 & 16,6 & 5 & 16,6 & 10 & 33,3 \\
\hline & Ed. Social & 1 & 3,3 & 1 & 3,3 & 2 & 6,6 \\
\hline & Otros & 2 & 6,6 & 6 & 20 & 8 & 26,6 \\
\hline & Total & 12 & 40 & 18 & 60 & 30 & 100 \\
\hline & $>26$ & 3 & 10 & 1 & 3,3 & 4 & 13,3 \\
\hline & $21-25$ & & & & & & \\
\hline & $16-20$ & & & 4 & 13,3 & 4 & 13,3 \\
\hline Experiencia & $11-15$ & 2 & 6,6 & 3 & 10 & 5 & 16,6 \\
\hline Protesional (anos) & $6-10$ & 3 & 10 & 1 & 3,3 & 4 & 13,3 \\
\hline & $<5$ & 4 & 13,3 & 9 & 30 & 13 & 43,3 \\
\hline & Total & 12 & 40 & 18 & 60 & 30 & 100 \\
\hline
\end{tabular}

Fuente: Elaboración propia.

Cuadro 3. Grupo de jueces para valorar escalas de claridad y adecuación

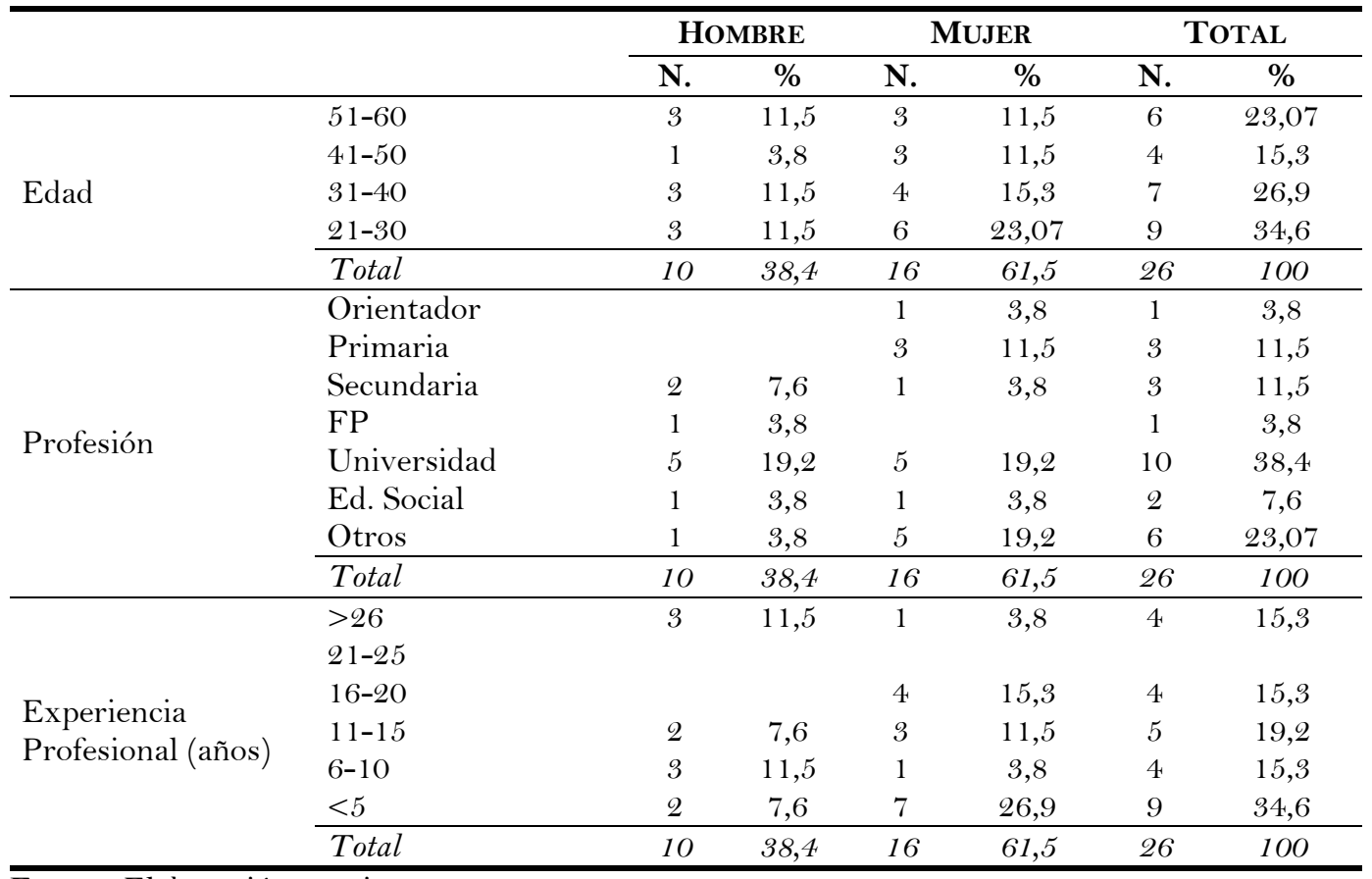

Fuente: Elaboración propia.

\subsection{Instrumentos}

Para el desarrollo de las diversas fases de consulta a expertos, se ha procedido según se describe en Jornet, González-Such y Bakieva (2017). El análisis documental se ha tomado como insumo para el ajuste inicial de la definición del constructo, sustentando el trabajo 
sobre un grupo focal o comité total de jueces expertos. Las valoraciones realizadas por comités de juicio se han estructurado en cada caso (indicadores de la definición e ítems) en escalas de valoración tipo Likert, de 4 puntos. Las escalas de valoración a considerar han sido: relevancia (importancia en la definición del constructo), susceptibilidad de cambio (grado en que el aspecto medido es susceptible de cambio mediante una intervención educativa orientada a la mejora), claridad de la formulación (de los ítems en relación a la población objeto de análisis), y adecuación (grado en que la formulación de los ítems es pertinente al problema y situación educativa).

De este modo, para el trabajo con los comités de juicio se ha desarrollado un cuestionario que incluye: datos personales, sin identificación nominal, los indicadores/ítems a valorar, según los cuatro criterios mencionados anteriormente, e ítems abiertos para recoger las propuestas de reformulación, observaciones o indicaciones acerca de posibles sesgos en la formulación (en total, $28+6=34$ ítems).

\subsection{Análisis de la información}

Para el análisis de la información se ha procedido inicialmente, a nivel teórico, al análisis de contenido. Para el estudio derivado de los comités de juicio se ha trabajado los diferentes elementos valorados mediante los análisis descriptivos; y para el análisis de la consistencia/congruencia inter-jueces se ha llevado a cabo el coeficiente W de Kendall y los valores resultantes de la correlación intra-clase. Asimismo, finalmente, se ha procedido a un análisis de conglomerados de K- medias para identificar perfiles de juicio, en relación con los criterios de valoración utilizados por los jueces.

\section{Resultados}

La presentación de resultados la organizamos en los siguientes apartados: bases teóricas, congruencia y consistencia inter-jueces (fiabilidad de juicio), características de los indicadores o ítems según los comités y análisis de conglomerados de K-medias.

\subsection{Bases teóricas}

Respecto al trabajo con el grupo focal, las categorías de formulación de indicadores que se tomaron de manera inicial se presentan en el cuadro 4.

La operativización de estas categorías para poder formular una escala aplicable, y, por lo tanto, comprensible, para el alumnado de educación primaria y secundaria obligatoria, dirigió la formulación de los ítems que posteriormente fueron valorados por los comités de expertos. Entre ambas dimensiones, inicialmente se identificaron 28 indicadores/elementos, 11 pertenecen a la primera dimensión y 17 a la segunda, establecidos ya en Jornet, Sancho y Bakieva (2015a), de los que prácticamente no han sufrido ninguna variación destacable). De este modo, con el fin de no ser reiterativos, tras los análisis realizados la traducción de los indicadores en ítems únicamente implicó la formulación de éstos al estilo de pregunta en un lenguaje adaptado a la población objeto. De esta manera, omitimos el listado de indicadores posterior y presentamos directamente los ítems tal y como quedaron diseñados previo y posterior al proceso de valoración de jueces (cuadros 6, 7 y 8). Por ello, téngase en cuenta que los ítems que se vayan a destacar a continuación en el proceso de valoración de jueces corresponden a los ítems reflejados en estos cuadros. Concretamente, su correspondiente traducción se realiza teniendo en cuenta que todos ellos son ítems de redacción previa a la valoración de jueces (es decir, en 
los cuadros 6 y 8 quedan indicados en la columna de la parte izquierda). Recordamos que el total de los ítems para esta fase es de 28 (a los que después se les suma 6 ítems más de carácter cualitativo).

Cuadro 4. Categorías de definición de ítems

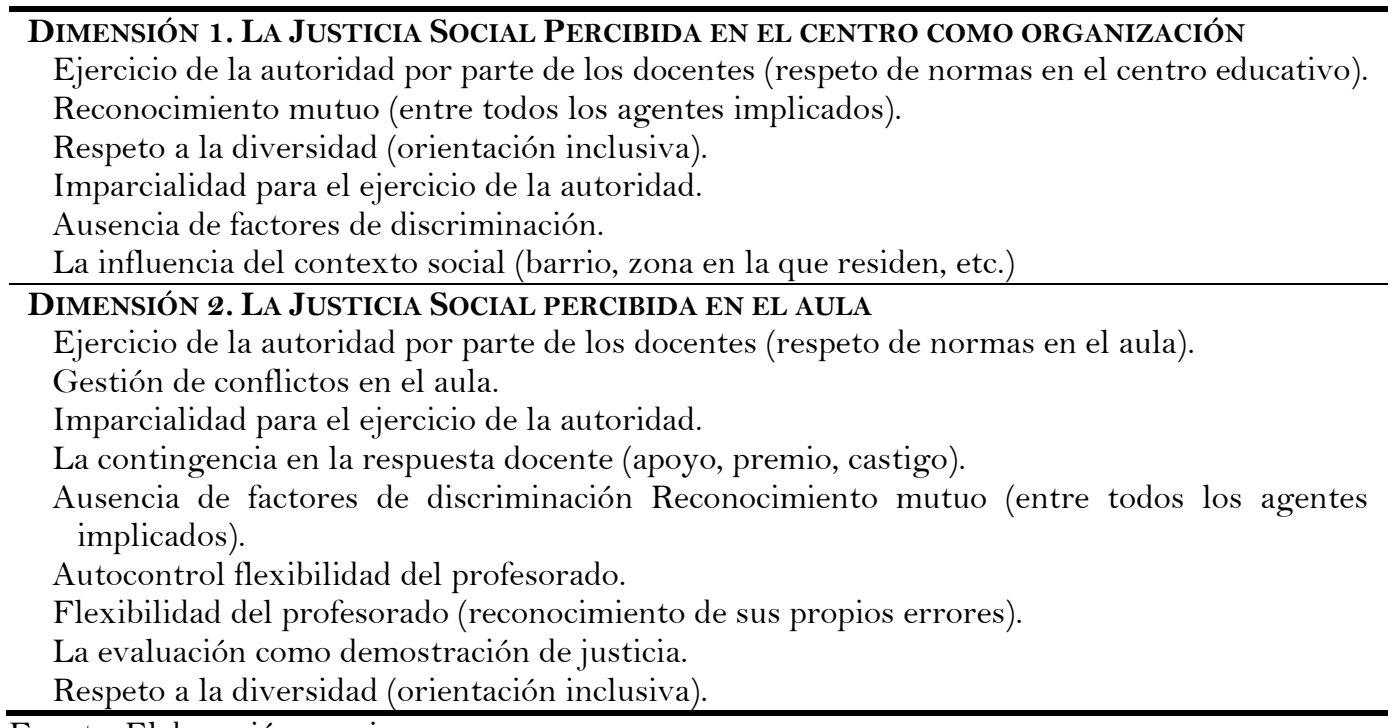

Fuente: Elaboración propia.

\subsection{Valoración de jueces: Congruencia y consistencia inter-jueces}

A continuación, hemos llevado a cabo el proceso de valoración de los jueces. Las opciones de valoración de las escalas (figura 2) se han presentado en una escala Likert de 4 opciones, siendo la opción 1 "nada" y la opción 4 "mucho". De las valoraciones obtenidas, hemos analizado los indicadores de congruencia y consistencia. Estos indicadores se han tomado como garantía para la selección posterior de elementos, buscando así un consenso intersubjetivo de jueces. Y, posteriormente, de los criterios que se establezcan en este consenso saldrá la propuesta piloto. Por lo que refiere a la valoración de jueces que hemos llevado a cabo, en Jornet, González-Such y Bakieva (2017) comentamos la conveniencia de realizar análisis con procesos de juicio de forma iterativa de manera que se pueda identificar si en los comités (grupos totales) existen grupos de jueces de opinión concordante (grupo depurado) o de si se dan divergencias asociadas a subgrupos (grupo diferencial). De todos ellos hemos obtenido también los valores de congruencia y consistencia. En definitiva y para todos los casos, aportamos como indicador de consistencia la Correlación Intra-clase y para congruencia el coeficiente $\mathrm{W}$ de Kendall. El objetivo con dichas valoraciones es comprobar si se aportan resultados coherentes entre las diversas perspectivas (grupo total, depurado y diferencial) como evidencia de validación.

En el primer análisis, respecto a las escalas de relevancia y susceptibilidad de cambio (cuadro 5), vemos que la fiabilidad obtenida para el comité total en ambos casos es mediabaja. En el comité depurado sube en ambos casos, sobre todo en el caso de relevancia, aunque los resultados respecto a la escala de susceptibilidad total respecto al comité diferencial y sobre fiabilidad son los más óptimos. Observamos que en todos los casos al menos obtenemos significación de al menos 0,05 (*). Respecto al indicador de congruencia, observamos que en ambos comités depurados el resultado es más alto que la correlación intra-clase y que en todos los casos obtiene una significación asintótica bilateral significativa del 0,001 (***) lo que significa que existe acuerdo. En el comité depurado, 
mejora su puntuación de manera que se eleva y mantiene su significación. Sin embargo, en el grupo diferencial de la escala de relevancia observamos que baja su puntuación y así mismo pierde también la significación, mientras que en la escala de susceptibilidad mantiene estos valores de manera muy similar a los del comité depurado. Sin ninguna duda, los resultados más óptimos se encuentran en el grupo diferencial de la escala de susceptibilidad de cambio.

Cuadro 5. Indicadores de congruencia y consistencia intra-jueces de relevancia y susceptibilidad de cambio

\begin{tabular}{|c|c|c|c|c|c|c|c|c|}
\hline \multicolumn{9}{|c|}{ RELEVANCIA (FASE 1, COMITÉ 1, N=30) } \\
\hline \multicolumn{3}{|c|}{ COMITÉ TOTAL $(\mathrm{N}=30)$} & \multicolumn{3}{|c|}{$\begin{array}{c}\text { COMITÉ DEPURADO }(\mathrm{N}= \\
12)\end{array}$} & \multicolumn{3}{|c|}{ DIFERENCIALES $(\mathbf{N}=18)$} \\
\hline $\begin{array}{c}\mathbf{R} \\
\text { Intra- } \\
\text { clase }\end{array}$ & $\begin{array}{c}\text { W } \\
\text { Kendall }\end{array}$ & $\chi^{2}$ & $\begin{array}{c}\text { R } \\
\text { Intra- } \\
\text { clase }\end{array}$ & $\begin{array}{c}\text { W } \\
\text { Kendall }\end{array}$ & $\chi^{2}$ & $\begin{array}{c}\mathbf{R} \\
\text { Intra- } \\
\text { clase }\end{array}$ & $\begin{array}{c}\text { W } \\
\text { Kendall }\end{array}$ & $\chi^{2}$ \\
\hline $0,42^{* * * *}$ & $0,58^{* * *}$ & $473,616^{\text {** }}$ & $0,62^{* * * *}$ & $0,67^{* * * *}$ & $392,29^{* * * *}$ & $\mathrm{NE}$ & 0,39 & $77,45^{* *}$ \\
\hline \multicolumn{9}{|c|}{$\begin{array}{l}\text { SUSCEPTIBILIDAD DE CAMBIO (FASE 1, COMITÉ 1, N=30) } \\
\end{array}$} \\
\hline \multicolumn{3}{|c|}{ COMITÉ TOTAL $(\mathrm{N}=30)$} & \multicolumn{3}{|c|}{ COMITÉ DEPURADO $(\mathrm{N}=7)$} & \multicolumn{3}{|c|}{ DIFERENCIALES $(\mathrm{N}=23)$} \\
\hline $\begin{array}{c}R \\
\text { Intra- } \\
\text { clase } \\
\end{array}$ & $\begin{array}{c}\text { W } \\
\text { Kendall }\end{array}$ & $\chi^{2}$ & $\begin{array}{c}\mathbf{R} \\
\text { Intra- } \\
\text { clase } \\
\end{array}$ & $\begin{array}{c}\text { W } \\
\text { Kendall }\end{array}$ & $\chi^{2}$ & $\begin{array}{c}R \\
\text { Intra- } \\
\text { clase } \\
\end{array}$ & $\begin{array}{c}\text { W } \\
\text { Kendall }\end{array}$ & $\chi^{2}$ \\
\hline $0,37^{*}$ & $0,66^{* * * *}$ & $449,99^{*}$ & $0,46^{* *}$ & $0,65^{* * *}$ & $344,33^{* * * *}$ & $0,95^{* * *}$ & $0,64^{* * * *}$ & $166,44^{\text {***** }}$ \\
\hline
\end{tabular}

Nota: * significativo al 0,05. ** Significativo al 0,01*** Significativo al 0,001.

Fuente: Elaboración propia.

Respecto a los estadísticos de estas dos primeras escalas (cuadro 1 del anexo) observamos medias muy altas con respuestas muy homogéneas en la mayoría de los casos (teniendo en cuenta los resultados obtenidos en el cociente de variación, en lo sucesivo CV). El único juez que presenta respuestas más dispares es el juez 27 para la escala de relevancia. Los mayores resultados estadísticos obtenidos se encuentran en los jueces 7, 12, 16 y 20 para la escala de relevancia (con un 3,96) y en el juez 7 para la escala de susceptibilidad (con un 3,96 también). Las medias más bajas se encuentran en ambos casos en el juez 9 (con un 2,29).

En el segundo análisis, respecto a las escalas de claridad y adecuación (cuadro 6) vemos que la consistencia inter-jueces obtenida para el comité total en ambos casos es baja. En el comité depurado sube en ambos casos y, sobre todo, en el caso de adecuación, siendo éste el resultado más óptimo de consistencia de todas las escalas valoradas. Observamos también que sólo en la escala de claridad se obtiene una significación de 0.05 o del 95\% (*). Respecto al indicador de congruencia, observamos que en ambos comités depurados el resultado es más alto que la correlación intra-clase y que en todos los casos obtiene una significación asintótica bilateral significativa siendo en la escala de adecuación de 0,001 (***) y en la de claridad de 0,05 (*). Tanto en el comité depurado como en los diferenciales, el W de Kendall mantiene su puntuación de manera muy similar al comité total. Sin ninguna duda, los resultados más óptimos se encuentran en la escala de adecuación, tanto en el comité depurado como en el diferencial, donde además todos los valores obtienen significación del 0,001 (***) que denotaría la interpretación de un consenso mayor.

Respecto a los estadísticos de estas otras escalas (cuadro 2 del anexo) observamos de nuevo medias muy altas con respuestas muy homogéneas en la mayoría de los casos (teniendo en cuenta los resultados obtenidos en CV). Los mayores resultados estadísticos obtenidos se encuentran en el juez 7 para la escala de claridad (con un 3,96) y en los jueces 
13 y 18 para la escala de adecuación (con un 3,97). Las medias más bajas se encuentran en ambos casos en el juez 9 (con un 2,29).

Cuadro 6. Indicadores de congruencia y consistencia intra-jueces de claridad y adecuación

\begin{tabular}{|c|c|c|c|c|c|c|c|c|}
\hline \multicolumn{9}{|c|}{ ClaRIDAD (FASE 2, CoMité 1, N = 26) } \\
\hline \multicolumn{3}{|c|}{ COMITÉ TOTAL $(\mathrm{N}=26)$} & \multicolumn{3}{|c|}{ COMITÉ DEPURADO $(\mathbf{N}=8)$} & \multicolumn{3}{|c|}{ DIFERENCIALES $(\mathbf{N}=18)$} \\
\hline $\begin{array}{c}R \\
\text { Intra- } \\
\text { clase }\end{array}$ & $\begin{array}{c}\text { W } \\
\text { Kendall }\end{array}$ & $\chi^{2}$ & $\begin{array}{c}\mathbf{R} \\
\text { Intra- } \\
\text { clase }\end{array}$ & $\begin{array}{c}\text { W } \\
\text { Kendall }\end{array}$ & $\chi^{2}$ & $\begin{array}{c}R \\
\text { Intra- } \\
\text { clase }\end{array}$ & $\begin{array}{c}\text { W } \\
\text { Kendall }\end{array}$ & $\chi^{2}$ \\
\hline $0,34^{*}$ & $0,70^{*}$ & $490,99^{*}$ & $0,51^{* * *}$ & $0,73^{* * * *}$ & $410,59^{* * * *}$ & $\mathrm{NE}$ & $0,61^{* * * *}$ & $68,43^{*}$ \\
\hline \multicolumn{9}{|c|}{ ADECUACIÓN (FASE 2, COMITÉ 1, N=26) } \\
\hline \multicolumn{3}{|c|}{ COMité Total $(\mathbf{N}=26)$} & \multicolumn{3}{|c|}{ COMITÉ DEPURADO $(\mathrm{N}=9)$} & \multicolumn{3}{|c|}{ DIFERENCIALES $(\mathrm{N}=17)$} \\
\hline $\begin{array}{c}\mathrm{R} \\
\text { Intra- } \\
\text { clase }\end{array}$ & $\begin{array}{c}\text { W } \\
\text { Kendall }\end{array}$ & $\chi^{2}$ & $\begin{array}{c}\mathbf{R} \\
\text { Intra- } \\
\text { clase }\end{array}$ & $\begin{array}{c}\text { W } \\
\text { Kendall }\end{array}$ & $\chi^{2}$ & $\begin{array}{c}\mathrm{R} \\
\text { Intra- } \\
\text { clase }\end{array}$ & $\begin{array}{c}\text { W } \\
\text { Kendall }\end{array}$ & $\chi^{2}$ \\
\hline 0,28 & $0,64^{* * * *}$ & 449,99 & $0,96^{* * *}$ & $0,63^{* * * *}$ & $366,79^{* * * *}$ & $0,93^{* * * *}$ & $0,63^{* * * *}$ & $73,34^{\text {**** }}$ \\
\hline
\end{tabular}

Nota: * significativo al 0,05. ** Significativo al 0,01*** Significativo al 0,001.

Fuente: Elaboración propia.

En general, observamos que la mayoría de los jueces expertos en cuanto a sus valoraciones sobre relevancia, susceptibilidad, claridad y adecuación, obtienen medias elevadas, en torno a 2,29 hasta 3,97 y con un CV bastante homogéneo. Ello significa que los jueces han otorgado valoraciones similares en sus respuestas y que podemos fiarnos de las medias estadísticas, teniendo en cuenta que éstas son altas o muy altas. Además, aunque el criterio de consistencia de la correlación intra-clase sea bajo para algunos casos, al realizar el análisis de las puntuaciones sobre el nivel estadístico de W de Kendall observamos cómo el resultado sí que es elevado y donde también presenta significación de al menos un 95\%. Es por ello que de manera general se considera que los ítems son valorados de forma homogénea por el conjunto de jueces. Esto es positivo porque denota que todos presentan una opinión similar sobre los criterios trabajados.

\subsection{Análisis descriptivos de criterios: Revisiones por ítems}

De manera general podemos observar que los resultados de las medias de los ítems son muy altos. Respecto a la escala de relevancia (cuadro 3 del anexo) el ítem con mayor media es el número $18(3,77)$ y el ítem con menor media es el número $4(3,4)$. Las opiniones de los jueces, teniendo en cuenta el $\mathrm{CV}$, son muy homogéneas. Ello quiere decir que existe bastante acuerdo a la hora de valorar. Las valoraciones más altas, cuando separamos grupos, las encontramos en el grupo de depurados respecto a los diferenciales, observándose que en los diferenciales se da mayor variabilidad si tenemos en cuenta el CV. Es decir, en el grupo de depurados se presenta mayor valoración a la vez que mayor consenso. Pese con todo, en ambos grupos (depurados y diferenciales) las valoraciones son elevadas. Además, al realizar la U de Mann Whitney observamos que no existen diferencias estadísticamente significativas entre ambos grupos y en ningún ítem, por lo que podemos asumir que la valoración del grupo total es representativa.

Respecto a la escala de susceptibilidad de cambio (cuadro 4 del anexo) los ítems con mayor media son el número 18 y el 25 (3,73) y el ítem con menor media es el número 17 (3,33). Las opiniones de los jueces, teniendo en cuenta el CV, son también muy homogéneas. Las valoraciones más altas, cuando separamos grupos, las encontramos en el grupo de diferenciales respecto a los depurados, aunque observándose que en los diferenciales se da mayor variabilidad si observamos el CV. Es decir, en el grupo de diferenciales se presenta 
mayor valoración, pero no obtienen un mayor consenso. Aun así, en ambos grupos las valoraciones son elevadas. Además, al realizar la U de Mann Whitney observamos que tampoco existen diferencias estadísticamente significativas entre ambos grupos y en ningún ítem. De nuevo podemos asumir que la valoración del grupo total es suficientemente representativa.

En lo que refiere a la escala de claridad (cuadro 5 del anexo) los ítems con mayor media son el número $18(3,77)$ y el ítem con menor media es el número 17 (3,35). Las opiniones de los jueces, teniendo en cuenta el $\mathrm{CV}$, son nuevamente muy homogéneas. Las valoraciones más altas, cuando separamos grupos, las encontramos esta vez en el grupo de depurados respecto a los diferenciales donde, además, si tenemos en cuenta el CV, es donde también obtenemos mayor consenso. De igual manera, ambos grupos presentan valoraciones elevadas. Una vez más, al realizar la U de Mann Whitney observamos que no existen diferencias estadísticamente significativas entre ambos grupos y en ningún ítem. Nuevamente podemos asumir que la valoración del grupo total es representativa.

Respecto a la escala de adecuación (cuadro 6 del anexo) los ítems con mayor media son el número 18 (3,77) y el ítem con menor media es el número 7 (3,42). Las opiniones de los jueces, teniendo en cuenta el $\mathrm{CV}$, son también muy homogéneas. Las valoraciones más altas, cuando separamos grupos, las encontramos en el grupo de depurados respecto a los diferenciales, sin embargo, si tenemos en cuenta el CV es donde obtenemos menor consenso. Pese a todo, en ambos grupos las valoraciones son elevadas. Además, al realizar la U de Mann Whitney observamos que no existen diferencias estadísticamente significativas entre ambos grupos, pero sí respecto a algunos ítems. Encontramos diferencias significativas en el 95\% en los ítems 13, 14, 19 y 20. Aun así y con todo, podemos asumir que la valoración del grupo total es también suficientemente representativa.

\subsection{Análisis de conglomerados de K-medias}

Finalmente, para presentar de forma más precisa las puntuaciones más extremas de los grupos valorados, se realiza el análisis de conglomerados k-medias. Este análisis pretende concretar en torno a qué puntuaciones se concentran las puntuaciones diferenciales y a la vez pretende explorar cual es el número óptimo de grupos que se puede proponer para el diseño y validación del instrumento con el que pretendemos evaluar la JSP-E. En este sentido, los resultados obtenidos destacan que la propuesta más óptima se debe llevar a cabo con 2 grupos (resultados en el cuadro 7).

Además, la figura 4 ofrece los dos centros de conglomerados finales, es decir, los centros de conglomerados tras el proceso de iteración. La figura resume los valores centrales de cada conglomerado, observándose valores promedios de percepción por parte de los participantes por encima de 3 para el primer conglomerado, es decir, los participantes están entre el "muy de acuerdo" y "totalmente de acuerdo" con respecto a la escala Likert utilizada. Sin embargo, en los resultados del segundo conglomerado vemos que la respuesta oscila entre el "bastante de acuerdo" y "muy de acuerdo". La interpretación del primer conglomerado es que los expertos acogen de manera positiva el instrumento atendiendo a las diferentes dimensiones que se han propuesto. Por otro lado, el segundo conglomerado indica que la recogida de información sobre la percepción de los alumnos de justicia social puede, a través de las diferentes dimensiones, llegar a ser más baja. Estos resultados se interpretan de esta manera, pero hay que tener en cuenta que no llegan a ser significativos. 
Cuadro 7. Conglomerados de K-medias

\begin{tabular}{lccc}
\hline & \multirow{2}{*}{$\begin{array}{c}\text { No DEL } \\
\text { CONGLOMERADo }\end{array}$} & \multicolumn{2}{c}{ CLÚSTER DE K MEDIAS (CONGLOMERADO DE K } \\
\cline { 3 - 4 } & & MEDIAS) & 2 clúster \\
\cline { 3 - 4 } Relevancia & 1 & 19 & 19 \\
(30 jueces) & 2 & 11 & 10 \\
& 3 & - & 1 \\
\hline \multirow{2}{*}{ Susceptibilidad } & 1 & 22 & 7 \\
(30 jueces) & 2 & 8 & 1 \\
\hline \multirow{2}{*}{ Adecuación } & 3 & - & 20 \\
(26 jueces) & 1 & 20 & 5 \\
& 2 & 6 & 20 \\
\hline \multirow{2}{*}{ Claridad } & 3 & - & 5 \\
(26 jueces) & 1 & 20 & 1 \\
\hline
\end{tabular}

Fuente: Elaboración propia.

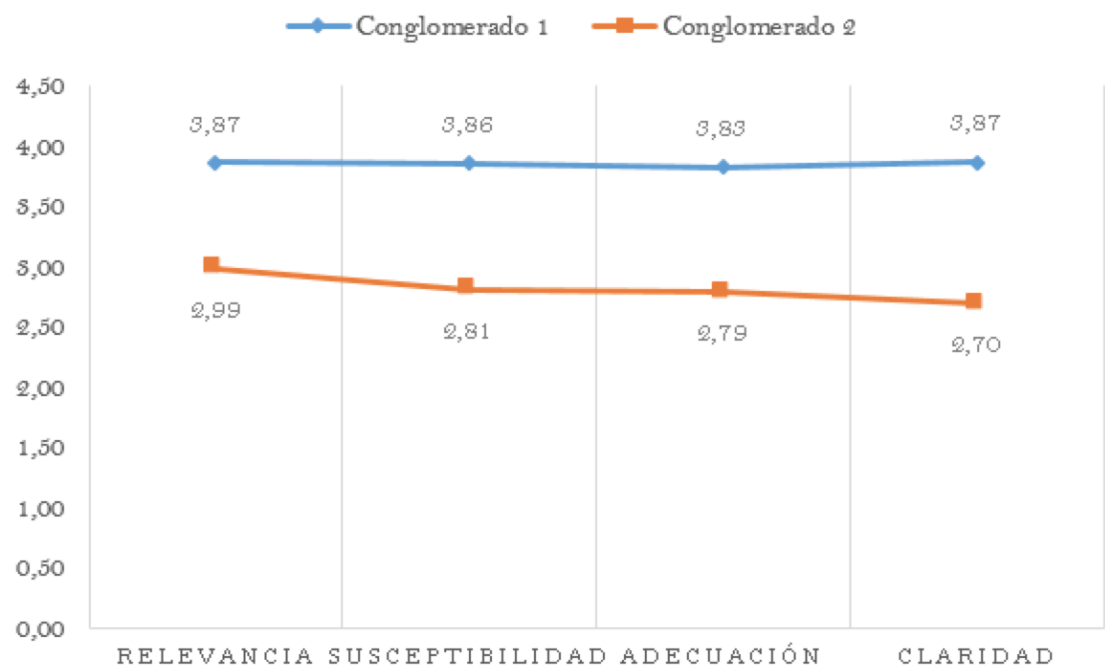

Figura 4. Centro de conglomerados finales

Fuente: Elaboración propia.

\subsection{Instrumento final}

En definitiva, todos los ítems han sido valorados de manera muy homogénea por el conjunto de jueces en todos los criterios que se han considerado en la revisión. Obsérvese que todos los cocientes de variación son inferiores al 33\% y, en muchos casos, no llegan tan siquiera al 20\%. Ello constituye una evidencia acerca de que los jueces han valorado cada elemento, respecto a todos los criterios trabajados, de forma muy similar por lo que las medias de cada reactivo pueden entenderse como muy representativas. El resultado obtenido es muy positivo ya que indica que por lo general hay una buena valoración de los criterios propuestos y ello es lo que nos va a permitir que podamos continuar diseñando nuestra propuesta. La versión final del instrumento se presenta a continuación. En los cuadros 8 y 10, se muestra una comparativa entre la propuesta previa a la valoración de jueces y cómo queda dicha propuesta tras la valoración. 
Cuadro 8. Comparativa instrumento previo y final a la valoración de jueces. Dimensión 1: La Justicia Social Percibida en el centro como organización

\begin{tabular}{|c|c|}
\hline PREVIO A LA VALORACIÓN DE LOS JUECES & $\begin{array}{l}\text { POSTERIOR A LA VALORACIÓN DE LOS } \\
\text { JUECES }\end{array}$ \\
\hline $\begin{array}{l}\text { 1. Las personas que dirigen el cole hacen que } \\
\text { se respeten las normas de convivencia. }\end{array}$ & $\begin{array}{l}\text { 1.1. ¿Las personas que dirigen el colegio } \\
\text { hacen que se respeten las normas de } \\
\text { convivencia? }\end{array}$ \\
\hline $\begin{array}{l}\text { 2. Los maestros/as de tu cole te ayudan a } \\
\text { solucionar las peleas que tenéis entre } \\
\text { compañeros/as. }\end{array}$ & Eliminado \\
\hline $\begin{array}{l}\text { 3. Crees que en tu colegio todos los/as } \\
\text { maestros/as son igual de importantes. }\end{array}$ & $\begin{array}{l}\text { 1.2. ¿Crees que en tu colegio todos los } \\
\text { maestros son igual de importantes? }\end{array}$ \\
\hline $\begin{array}{l}\text { 4. Has visto a maestros/as que rechazan a } \\
\text { niños/as por ser de otra religión, etnia o } \\
\text { país. }\end{array}$ & $\begin{array}{l}\text { 1.3. ¿Has visto a maestros que rechazan a } \\
\text { niños por ser de otra religión o de otro } \\
\text { país? }\end{array}$ \\
\hline $\begin{array}{l}\text { 5. Crees que hay maestros/as que tienen a } \\
\text { alumnos/as favoritos/as. }\end{array}$ & Eliminado \\
\hline $\begin{array}{l}\text { 6. El colegio permite que tus compañeros/as } \\
\text { te acepten tal y como eres. }\end{array}$ & $\begin{array}{l}\text { 1.4. ¿Los maestros permiten que tus } \\
\text { compañeros te acepten tal y como eres }\end{array}$ \\
\hline $\begin{array}{l}\text { 7. Hay maestros/as que no quieres que te den } \\
\text { clase porque son demasiado exigentes. }\end{array}$ & Eliminado \\
\hline $\begin{array}{l}\text { 8. Crees que el colegio trata mejor a unas } \\
\text { familias que a otras (porque tienen más } \\
\text { dinero, porque son de otro país o porque } \\
\text { tienen más estudios) }\end{array}$ & $\begin{array}{l}\text { 1.7. ¿Crees que el colegio trata mejor a unas } \\
\text { familias que a otras (porque tienen más } \\
\text { dinero, porque son de otro país o porque } \\
\text { tienen más estudios)? }\end{array}$ \\
\hline $\begin{array}{l}\text { 9. Los/as maestros/as dan la atención } \\
\text { necesaria a los alumnos/as en el patio. }\end{array}$ & $\begin{array}{l}\text { Reformulado: } 1.8 \cdot \text { ¿Los maestros están } \\
\text { pendientes de todos los alumnos para que } \\
\text { no haya problemas entre ellos (en el patio, } \\
\text { en los pasillos)? }\end{array}$ \\
\hline $\begin{array}{l}\text { 10. Los/as maestros/as ayudan a los/as } \\
\text { alumnos/as que tienen más dificultades. }\end{array}$ & Eliminado \\
\hline $\begin{array}{l}\text { 11. Los/as maestros/as hablan con los/as } \\
\text { alumnos/as de manera individual cuando } \\
\text { alguien lo necesita. }\end{array}$ & Eliminado \\
\hline
\end{tabular}

Fuente: Elaboración propia.

En el cuadro 9 se presentan otros ítems que han surgido durante el proceso de reflexión. Los jueces han estimado oportuno añadirlos tras las modificaciones o eliminaciones realizadas o simplemente por el hecho de que el instrumento no había tenido en cuenta, en su forma previa, criterios similares a ellos.

Cuadro 9. Ítems que han surgido durante el proceso de valoración y que se añaden después al instrumento

1.5. ¿Hay maestros que te riñen demasiado?

1.6. ¿Hay maestros que no quieres que te den clase porque no te tratan bien?

Fuente: Elaboración propia.

\section{Discusión y conclusiones}

Respecto al diseño del instrumento, dentro del marco de discusión académica, hemos tenido en cuenta otras escalas que han trabajado las consideraciones de la justicia social percibida en el ámbito educativo (cuadro 1). Para ello, y principalmente, nos hemos centrado en las aportaciones de las investigaciones de Ludlow, Enterline y Cochran-Smith 
(2008) y Jacott y otros (2014), las cuales desarrollan cómo la percepción de los agentes educativos puede llegar a ser fiable para medir la JSP-E dentro del ámbito escolar.

Cuadro 10. Comparativa instrumento previo y final a la valoración de jueces. Dimensión 2: La Justicia Social Percibida en el aula

\begin{tabular}{|c|c|}
\hline PREVIO A LA VALORACIÓN DE LOS JUECES & POSTERIOR A LA VALORACIÓN DE LOS JUECES \\
\hline $\begin{array}{l}\text { 12. Crees que tus maestros/as tienen } \\
\text { alumnos/as preferidos/as. }\end{array}$ & $\begin{array}{l}\text { 2.1. ¿Crees que tus maestros tienen alumnos } \\
\text { preferidos? }\end{array}$ \\
\hline $\begin{array}{l}\text { 13. Crees que tus maestros/as tienen manía a } \\
\text { algunos/as alumnos/as. }\end{array}$ & $\begin{array}{l}\text { 2.2. ¿Crees que tus maestros tienen manía a } \\
\text { algunos alumnos? }\end{array}$ \\
\hline $\begin{array}{l}\text { 14. Crees que tus maestros/as de clase te } \\
\text { tratan de la misma manera a ti que al resto } \\
\text { de tus compañeros/as. }\end{array}$ & $\begin{array}{l}\text { 2.3. ¿Crees que tus maestros de clase te tratan } \\
\text { de la misma manera a ti que al resto de tus } \\
\text { compañeros o compañeras? }\end{array}$ \\
\hline $\begin{array}{l}\text { 15. Tus maestros/as son justos/as cuando } \\
\text { solucionan problemas entre alumnos/as. }\end{array}$ & $\begin{array}{l}\text { 2.4. ¿Son tus maestros justos cuando } \\
\text { solucionan problemas entre alumnos? }\end{array}$ \\
\hline $\begin{array}{l}\text { 16. Tus maestros/as se enfadan si los/as } \\
\text { alumnos/as se equivocan. }\end{array}$ & $\begin{array}{l}\text { 2.5. ¿Se enfadan si los alumnos os equivocáis } \\
\text { en clase? }\end{array}$ \\
\hline $\begin{array}{l}\text { 17. Crees que tus maestros/as crean normas } \\
\text { de clase injustas. }\end{array}$ & $\begin{array}{l}\text { 2.6. ¿Crees que tus maestros crean normas de } \\
\text { clase injustas? }\end{array}$ \\
\hline $\begin{array}{l}\text { 18. Tus maestros/as intentan ayudarte } \\
\text { cuando tienes problemas. }\end{array}$ & $\begin{array}{l}\text { 2.7. ¿Intentan ayudarte tus maestros cuando } \\
\text { tienes problemas? }\end{array}$ \\
\hline $\begin{array}{l}\text { 19. Crees que te mereces las notas que te } \\
\text { ponen tus maestros/as. }\end{array}$ & $\begin{array}{l}\text { 2.8. ¿Crees que te mereces las notas que te } \\
\text { ponen tus maestros? }\end{array}$ \\
\hline $\begin{array}{l}\text { 20. Crees que compañeros/as de tu clase } \\
\text { aprueban sin merecérselo. }\end{array}$ & Eliminado \\
\hline $\begin{array}{l}\text { 21. Tus maestros/as ponen mejores notas a } \\
\text { los alumnos/as que les caen bien. }\end{array}$ & $\begin{array}{l}\text { 2.9. ¿Crees que tus maestros ponen mejores } \\
\text { notas a los alumnos que les caen bien? }\end{array}$ \\
\hline $\begin{array}{l}\text { 22. Piensas que cualquiera de tus } \\
\text { maestros/as, aunque te conozca poco, te } \\
\text { felicita cuando haces algo bien. }\end{array}$ & $\begin{array}{l}\text { Reformulado: } 2.10 . ¿ \text { ¿Te felicitan tus maestros } \\
\text { cuando te portas bien? }\end{array}$ \\
\hline $\begin{array}{l}\text { 23. Piensas que cualquiera de tus } \\
\text { maestros/as, aunque te conozca poco, te } \\
\text { felicita cuando trabajas bien. }\end{array}$ & $\begin{array}{l}\text { Reformulado: } 2.11 . ¿ \text { ¿Te felicitan tus maestros } \\
\text { cuando trabajas bien? }\end{array}$ \\
\hline $\begin{array}{l}\text { 24. Tus maestros/as reconocen sus propios } \\
\text { errores en clase. }\end{array}$ & $\begin{array}{l}\text { 2.12. ¿Tus maestros reconocen sus propios } \\
\text { errores en clase? }\end{array}$ \\
\hline $\begin{array}{l}\text { 25. Tus maestros/as te ayudan a entender lo } \\
\text { que es justo y lo que es injusto. }\end{array}$ & $\begin{array}{l}\text { Reformulado: } \\
\text { 2.13. ¿Te ayudan tus maestros a entender lo } \\
\text { que es justo y lo que es injusto? (te explican } \\
\text { la diferencia) } \\
\text { 2.14. ¿'Tus maestros tratan en clase lo que es } \\
\text { justo y lo que es injusto? }\end{array}$ \\
\hline $\begin{array}{l}\text { 26. Crees que hay injusticias en tu barrio que } \\
\text { te afectan. }\end{array}$ & \multirow{2}{*}{$\begin{array}{l}\text { Reformulado: } \\
\text { 1.9. ¿Crees que hay injusticias en tu barrio o } \\
\text { zona donde vives que te afectan (violencia, } \\
\text { drogas, pobreza)? }\end{array}$} \\
\hline $\begin{array}{l}\text { 27. Crees que hay injusticias en el mundo } \\
\text { que te afectan. }\end{array}$ & \\
\hline $\begin{array}{l}\text { 28. Tus maestros/as te animan a participar } \\
\text { en las actividades de clase. }\end{array}$ & $\begin{array}{l}\text { 2.15. ¿Tus maestros os animan a participar en } \\
\text { las actividades de clase? }\end{array}$ \\
\hline
\end{tabular}

Fuente: Elaboración propia.

Los últimos autores, por ejemplo, ya destacaban la escasez de estudios cuantitativos que trabajasen las representaciones de los estudiantes y de los profesores acerca de la justicia social y respecto a las implicaciones que ésta puede tener dentro del proceso evaluativo (Hidalgo, 2017). De manera diferente a la nuestra, ya que nosotros proponemos el diseño de un cuestionario, Jacott y otros (2014) presentan un instrumento basado en dilemas. Sin embargo, y al igual que nosotros, lo desarrollan a partir de la teoría de justicia social para el ámbito educativo de Fraser (2008), teoría considerada dentro de la escuela crítica y 
nuevas teorías del siglo XX y XXI (clasificación realizada por Llorens, 2016). Así pues, ellos diseñan ítems relacionados con las tres dimensiones de dicha teoría: la distribución, el reconocimiento y la representación. En nuestro trabajo destacamos que la dimensión más reflexionada ha sido la de reconocimiento y dentro de ella hemos trabajado sobre todo la consideración de llevar a cabo una evaluación justa (Hidalgo, 2017) que refleje la existencia o inexistencia de equidad de oportunidades y reconocimiento de las acciones que el profesorado lleva a cabo con el alumnado. En concreto, en el estudio de Jacott y otros (2014) se mencionan los resultados obtenidos, pero no especifican de manera pormenorizada el procedimiento de creación del instrumento o al menos, no de una manera en que nosotros podamos realizar comparaciones. Sin embargo, para nosotros ha sido importante dar a conocer el número de jueces que han valorado cada una de las escalas al igual que hemos querido reflejar su experiencia profesional y académica, la paridad en el sexo y otras variables interesantes como es la edad. Estos datos los hemos querido dar a conocer para poder comprobar, en futuros estudios, si dichas variables han podido influir y de qué manera en las respuestas de los jueces. En esta línea, una de las pocas consideraciones que se explicitan en Jacott y otros (2014) son los orígenes académicos de los expertos que han participado en su estudio. Tanto en su propuesta como en la nuestra, vemos que los resultados métricos son muy positivos y ello lo demuestra la homogeneidad de respuestas que han llevado a cabo los jueces durante todo el proceso. Por otra parte, uno de los estudios que sí que ha explicitado de manera pormenorizada, al igual que nosotros, el proceso de validación de su instrumento de valoración (en concreto una escala de creencias) es el de Ludlow, Enterline y Cochran-Smith (2008), donde intentan demostrar cómo los análisis de TRI (Teoría de Respuesta al Ítem) pueden llegar a ser mucho más fiables que los de TCT (Teoría Clásica de los Tests) para el campo académico de las percepciones. Desde nuestra perspectiva, esta puede ser una de las líneas futuras de investigación a tener en cuenta. Además, y principalmente de manera singular, en este trabajo hemos querido destacar qué subgrupos de jueces se han formado para aportar mayor validez a nuestro instrumento, dividiendo así las respuestas más homogéneas en el grupo que hemos llamado "depurado" y las respuestas dispersas en el grupo que hemos llamado "diferencial".

Por otra parte, aunque el estudio de Ludlow, Enterline y Cochran-Smith (2008) va remitido al profesorado, tienen en cuenta algunos de los criterios que a nosotros también nos interesan, como son: las actitudes de respeto e inclusión por parte del profesorado hacia la multiculturalidad, si el profesorado ayuda o no a los educandos con dificultades, la responsabilidad de los equipos educativos por mantener hábitos educativos o de si existen relaciones justas en las instituciones. Este estudio lo hemos considerado fuertemente vinculado a nuestro interés de investigación ya que, ellos al igual que nosotros, pretenden fomentar una educación crítica que no sólo deba quedarse enmarcada dentro de la propia institución educativa sino también en la sociedad o en el contexto en el que vive el alumnado. De manera semejante a la nuestra, estos autores pretenden estudiar si las percepciones que se obtengan de su estudio pueden llegar a proyectarse en la personalidad futura de los participantes y de si ello pueda llegar a afectarles en las relaciones sociales futuras que les toque vivir.

Además, en nuestro trabajo los jueces han valorado las escalas de relevancia, susceptibilidad de cambio, claridad y adecuación. La consideración de dichos criterios en su conjunto se encuentra en muy pocos trabajos, puesto que sólo la podremos encontrar en aquellas escalas que enmarquen su instrumento dentro de la búsqueda de la Cohesión 
Social (Jornet, 2012). Por ejemplo, el criterio de susceptibilidad de cambio (educativo) lo hemos introducido como un aspecto inherente al concepto de evaluación en el sentido de cómo pueden influir las percepciones del alumnado en el proceso educativo y cómo éstas pueden orientar al cambio o la mejora de dicho proceso. Éste es el sentido de equidad y mejora educativa que previamente se ha estudiado y desarrollado en estudios como Jornet (2012) o Hidalgo (2017).

En lo que se refiere a las conclusiones, realizaremos una reflexión global para evitar ser reiterativos y no volver a desarrollar las preocupaciones ya tratadas en otros apartados. En concreto, respecto a las características de nuestro instrumento, hemos tenido en cuenta las implicaciones que puede tener la Educación para trabajar la Justicia Social (Fraser, 2008; Hidalgo, 2017; Jornet, Sancho y Bakieva, 2015b; Murillo y Hernández, 2011, entre otros) y de ahí hemos pasado a desarrollar nuestra propia propuesta. A partir de los intereses educativos que tratan dichos autores en sus respectivos estudios, hemos tenido en cuenta cuáles de éstos podían ser los más próximos a nuestra propuesta de JSP-E y los hemos reflejado en el instrumento, considerando algunos tales como el aprendizaje colaborativo, crítico y reflexivo, y otras valoraciones que tengan que ver con la motivación del alumnado, su rendimiento (Bravo et al., 2008), su inclusión, su respeto, sus emociones y opiniones, etc.

Respecto a las valoraciones emitidas por los jueces, vemos que los resultados métricos son óptimos. Por ejemplo, la escala de susceptibilidad muestra que los jueces entienden que todos los aspectos valorados por los ítems son susceptibles de cambio, es decir, que podríamos orientar programas de intervención educativa para su mejora. En general, y para todas las escalas, existe acuerdo entre los expertos respecto a los criterios analizados y esto es un aval para pensar que estamos en una buena dirección al traducir la definición teórica en ítems, es decir, al operativizar el constructo en un instrumento de medida (una escala, en concreto, sería la traducción de nuestra propuesta). Hay que destacar que las medias obtenidas en el proceso de valoración han sido muy altas, pues ninguna baja de 3 puntos respecto a una escala de Likert de 1 a 4 puntos. El ítem con media inferior en la mayoría de los casos es el ítem número 17 (Crees que tus maestros/as crean normas de clase injustas). De igual manera, si consideramos estos resultados comparándolos directamente con los análisis que hemos llevado a cabo para interpretar la consistencia inter-jueces del constructo (W de Kendall) obtenemos que son positivos. Únicamente, los resultados extraídos en el ítem 17 muestran que los jueces podrían pensar que el alumnado no va a poder valorar cómo el maestro puede transmitir las normas de clase porque quizá no siempre todas las reglas se hagan explícitas o no siempre se transmitan con total claridad. Aquí los jueces pueden haber estado asumiendo que el alumnado necesita un nivel cognitivo mayor para poder juzgar cada una de las normas de clase. Independientemente de este resultado, consideramos que este criterio puede seguir estando bien planteado ya que puede haber profesores o maestros que sí que dejen claras y explícitas cuáles vayan a ser las normas de clase y, por lo tanto, en este caso, la percepción podría ser más fácilmente identificable.

De manera general, para poder consensuar éstas y otras explicaciones relativas a los criterios finales hemos establecido el consenso intersubjetivo de jueces como base de objetividad teniendo en cuenta cada uno de los criterios valorados. Hay que destacar que durante el proceso de valoración no ha habido ninguna propuesta llamativa de cambio en la definición ni tampoco ninguna valoración negativa de sesgo. De esta manera, podemos 
argumentar que este proceso aporta una aproximación inicial de validez a nuestro futuro instrumento.

En definitiva, respecto al proceso de valoración, los jueces consideran que los ítems propuestos pueden llegar a medir las acciones del profesorado que, por parte del alumnado, puedan ser percibidas como justas. Es por ello que a partir de todas las propuestas de cambio que los jueces han establecido para cada uno de los ítems (cuadros 8, 9 y 10), concretamente la columna de la derecha-, se redactará un instrumento (una escala) que pueda llegar a ser utilizable para un futuro ensayo piloto.

Aun así y de manera concluyente, la Justicia Social Percibida o JSP-E requiere de un largo trabajo de estudio empírico y reflexivo. En ese sentido, nuestro trabajo no termina aquí. Consideramos que el instrumento aún puede depurarse mucho más. Asimismo, los análisis de propiedades métricas deben continuar, además, de que deben ser realizados sobre muestras de jueces representativas. Queda para el futuro también poder desarrollar un ensayo piloto que refleje las consideraciones de los propios estudiantes. Además, otra de las hipótesis futuras a comprobar sería si la percepción de justicia que van interiorizando los neófitos durante su proceso de enseñanza-aprendizaje podrá afectar a su consideración sobre las relaciones y la justicia en su vida social futura.

\section{Agradecimientos}

Este trabajo forma parte del Proyecto I+D financiado por el MINECO (España) "Sistema educativo y cohesión social: Diseño de un modelo de evaluación de necesidades" (Ref. Secs_Evalnec, $n^{\circ}$ 37437).

\section{Referencias}

APA., AERA. y NCME. (2014). Standards for educational and psychological tests and manuals. Washington, DC: American Psychological Association.

Bakieva, M. (2016). Diseño y validación de un instrumento para evaluar la Colegialidad Docente. Tesis doctoral. Universitat de València.

Barr, R. B. y Tagg, J. (1998). De la enseñanza al aprendizaje: Un nuevo paradigma para la educación de pregrado. Guadalajara: Universidad de Guadalajara.

Bloom, B., Englehart, M. Furst, E., Hill, W. y Krathwohl, D. (1956). Taxonomy of educational objectives: The classification of educational goals. Nueva York, NY: Longman.

Bravo, D., Falck, D., González, R., Manzi, J. y Peirano, C. (2008). La relación entre la evaluación docente y el rendimiento de los alumnos: evidencia para el caso de Chile. Santiago de Chile: Universidad de Chile.

Cochran-Smith, M., Ludlow, L., Ell, F., O'Leary, M. y Enterline, S. (2012). Learning to teach for social justice as a cross-cultural concept: Findings from three countries. European Journal of Educational Research, 1(2), 171-198. https://doi.org/10.12973/eu-jer.1.2.171

Connell, R.W. (1997). Escuelas y justicia social. Madrid: Ediciones Morata.

Fraser, N. (2008). Scales of justice. Reimagining political space in a globalizing world. Nueva York, NY: Columbia University Press.

Ginns, P., Fryer, L., Amazan, R., McCormick y Loughland, A. (2014). Evaluation of the learning to teach for social justice-beliefs scale in an Australian context. Higher Education Research \& Development, 95(3), 1-13. 
Hidalgo, N. (2017). Concepciones sobre una evaluación justa de los estudiantes. Una aproximación fenomenográfica. Tesis doctoral. Universidad Autónoma de Madrid.

Jacott, L., Maldonado, A., Sainz, V., Juanes, A., García-Vélez, T. y Seguro, V. (2014). Representations of social justice amongst Spanish teachers and students. En P. Cunningham y N. Fretwell (Eds.), Innovative practice and research trends in identity, citizenship and education (pp. 122-139). Londres: CiCe.

Jornet, J. M. (2012). Las dimensiones docentes y cohesión social: Reflexiones desde la evaluación. Revista Iberoamericana de Evaluación Educativa, 5(1), 349-362.

Jornet, J. M., González-Such, J. y Bakieva, M. (2017). Aproximaciones cualitativas para la definición y validación de constructos de instrumentos estandarizados de medida. Enfoque teórico y metodología. Comunicación presentada en el $6^{\circ}$ Congresso Ibero-Americano em investigação qualitativa and $2^{\circ}$ International Symposium on Qualitative Research. Portugal.

Jornet, J. M., Sancho, C. y Bakieva, M. (2015a). Diseño y validación del constructo teórico de justicia social percibida por el alumnado en los centros escolares. Revista Internacional de Educación para la Justicia Social, 4(2), 111-126.

Jornet, J. M., Sancho, C. y Bakieva, M. (2015b). Validación del constructo de justicia social percibida. Revista Iberoamericana de Evaluación Educativa, 4(1), 51-77.

Kelly, G. A. (1955). The psychology of personal constructs. Nueva York, NY: Norton.

Leyva, Y. (2004). Validez de constructo en la evaluación de competencias médicas mediante pruebas referidas al criterio. Tesis doctoral. Universidad Autónoma de Aguascalientes.

Llorens, A. J. (2016). Diseño de una escala para evaluar la justicia social percibida en la escuela. Trabajo fin de Máster. Valencia: Universitat de València.

Ludlow, L. H., Enterline, S. E. y Cochran-Smith, M. (2008). Learning to teach for social justice beliefs scale: An application of rasch measurement principles. Measurement and Evaluation in $\begin{array}{llll}\text { Counselling and Development, } & 40(4), & 194-211 .\end{array}$ https://doi.org/10.1080/07481756.2008.11909815

Lundgren, U. P. (2013). PISA como instrumento político. La historia detrás de la creación del programa PISA. Profesorado. Revista de Currículum y Formación del Profesorado, 17(2), 16-29.

Murillo, F. J. y Hernández-Castilla, R. (2011). Hacia un concepto de justicia social. REICE. Revista Iberoamericana sobre Calidad, Eficacia y Cambio en Educación, 9(4), 7-23.

Murillo, F. J. e Hidalgo, N. (2015). Enfoques fundamentantes de la evaluación de estudiantes para la justicia social. Revista Iberoamericana de Evaluación Educativa, 8(1), 43-61.

Rivas, F., Alcantud, F. y Gaya, C (1994). La técnica de rejilla: Manual de construcción, aplicación y tratamiento de la información. Valencia: Programa GOLIAT.

Ruiz-Primo, A., Jornet, J. M. y Backhoff, E. (2006). Acerca de la validez de los exámenes de la calidad y el logro educativos (Excale). Ciudad de México: Instituto Nacional de Evaluación Educativa.

Sancho, C., Jornet, J. M. y González-Such, J. (2016). El constructo valor social subjetivo de la educación: validación cruzada entre profesorado de escuela y universidad. Revista de Investigación Educativa, 34(2), 329-350. https://doi.org/10.6018/rie.34.2.226131

Syverson, M. (2009). Social justice and evidence-based assessment with the learning record. Forum on Public, Policy, 1(29), 5-27.

Torres-Harding, S. R., Steele, C., Schulz, E., Taha, F. y Pico, C. (2014). Student perceptions of social justice and social justice activities. Education, Citizenship E Social Justice, 1(9), 55-66. https://doi.org/10.1177/1746197914520655

UNESCO. (2012). Educación para el desarrollo sostenible. París: UNESCO. 


\section{Anexos}

Cuadro 1. Estadísticos de los elementos y total-elemento de relevancia y susceptibilidad

\begin{tabular}{|c|c|c|c|c|c|c|c|c|c|c|}
\hline \multirow[b]{2}{*}{ JUECES } & \multicolumn{3}{|c|}{ RELEVANCIA } & \multicolumn{3}{|c|}{ SUSCEPTIBILIDAD } & \multicolumn{2}{|c|}{ RELEVANCIA } & \multicolumn{2}{|c|}{ SUSCEPTIBILIDAD } \\
\hline & Med. & $\begin{array}{l}\text { Des. } \\
\text { típica }\end{array}$ & CV & Med. & $\begin{array}{l}\text { Des. } \\
\text { típica }\end{array}$ & $\mathbf{C V}$ & $\begin{array}{l}\text { Correla. elemento- } \\
\text { total corregida }\end{array}$ & $\begin{array}{l}\text { Alfa de Cronbach si se } \\
\text { elimina el elemento }\end{array}$ & $\begin{array}{l}\text { Correl. elemen- } \\
\text { total corregida }\end{array}$ & $\begin{array}{c}\text { Alfa de Cronbach si se } \\
\text { elimina el elemento }\end{array}$ \\
\hline Juez 1 & 3,89 & 0,31 & 8,09 & 3,79 & ,63 & 16,64 &, 11 & ,419 &,- 13 & ,425 \\
\hline Juez2 & 4,00 & 0,00 & 0,00 & 4,00 & 0,00 & 0,00 & 0,00 & 422 & 0,00 & ,367 \\
\hline Juez3 & 3,43 & 0,88 & 25,63 & 3,71 &, 60 & 16,15 &,- 08 & 481 &, 00 & ,386 \\
\hline Juez4 & 3,75 & 0,44 & 11,76 & 3,68 & ,61 & 16,63 &,- 27 & ,475 & ,OO & ,387 \\
\hline Juez5 & 3,00 & 0,00 & 0,00 & 3,00 & 0,00 & 0,00 & 0,00 & ,422 & 0,00 & ,367 \\
\hline Juez6 & 3,39 & 0,69 & 20,20 & 3,93 &, 38 & 9,62 &,- 08 & ,463 & ,38 & 309 \\
\hline Juez7 & 3,96 & 0,19 & 4,77 & 3,96 &, 19 & 4,77 &, 02 & 427 &, 43 & 334 \\
\hline Juez8 & 2,57 & 0,69 & 26,84 & 2,36 & ,62 & 26,37 &, 11 & 418 & ,26 & ,311 \\
\hline Juez9 & 2,29 & 0,85 & 37,38 & 2,29 & ,85 & 37,38 & ,62 & ,235 &, 15 & ,344 \\
\hline Juez 10 & 3,68 & 0,48 & 12,93 & 3,93 & ,26 & 6,68 &, 52 & ,340 &, 14 & ,358 \\
\hline Juez 11 & 3,93 & 0,26 & 6,68 & 3,93 & ,38 & 9,62 &,- 05 & , 435 &,- 29 &, 426 \\
\hline Juez 12 & 3,96 & 0,19 & 4,77 & 2,82 & 61 & 21,69 &,- 21 & ,443 &, 21 & ,327 \\
\hline Juez 13 & 2,96 & 0,19 & 6,38 & 2,96 &, 51 & 17,13 &,- 04 & ,431 &, 22 & ,330 \\
\hline Juez 14 & 4,00 & 0,00 & 0,00 & 4,00 & 0,00 & 0,00 & 0,00 & ,422 & 0,00 & ,367 \\
\hline Juez 15 & 3,14 & 0,65 & 20,70 & 3,68 &, 48 & 12,93 &, 27 & ,377 &, 43 &, 281 \\
\hline Juez 16 & 3,96 & 0,19 & 4,77 & 3,86 & ,36 & 9,24 & ,02 & 427 &, 11 & ,359 \\
\hline Juez 17 & 3,86 & 0,36 & 9,24 & 3,89 & 57 & 14,56 &,- 24 & 462 &, 10 & ,359 \\
\hline Juez 18 & $4, \mathrm{OO}$ & 0,00 & 0,00 & 4,00 & 0,00 & 0,00 & 0,00 & ,422 & 0,00 & ,367 \\
\hline Juez 19 & 4,00 & 0,00 & 0,00 & 4,00 & 0,00 & 0,00 & 0,00 & ,422 & 0,00 & ,367 \\
\hline Juez20 & 3,96 & 0,19 & 4,77 & 3,39 &, 50 & 14,66 & ,02 & 427 &,- 14 &, 413 \\
\hline Juez2 1 & 3,39 & 0,50 & 14,66 & 2,75 &, 52 & 18,84 &, 28 & ,385 & ,O 1 & ,382 \\
\hline Juez22 & 2,75 & 0,66 & 23,47 & 3,68 &, 48 & 12,93 &,$- \mathrm{OO}$ & , 444 &, 22 & ,331 \\
\hline Juez23 & $4, \mathrm{OO}$ & 0,00 & $\mathrm{O}, \mathrm{OO}$ & $4, \mathrm{OO}$ & 0,00 & $\mathrm{O}, \mathrm{OO}$ & 0,00 & ,422 & 0,00 & ,367 \\
\hline Juez24 & 4,00 & 0,00 & 0,00 & 4,00 & 0,00 & 0,00 & 0,00 & ,422 & 0,00 & ,367 \\
\hline Juez25 & 3,32 & 0,77 & 23,25 & 3,93 & ,26 & 6,68 &, 23 & ,383 &, 14 & ,358 \\
\hline Juez26 & 4,00 & 0,00 & 0,00 & 4,00 & 0,00 & 0,00 & 0,00 &, 422 & 0,00 & ,367 \\
\hline
\end{tabular}




\begin{tabular}{ccccccccccc}
\hline Juez27 & 3,11 & 1,13 & 36,47 & 3,89 &, 31 & 8,09 &, 24 &, 374 &, 18 \\
Juez28 & 3,64 & 0,49 & 13,39 & 3,64 &, 49 & 13,39 &, 19 &, 402 &, 350 \\
Juez29 & 3,07 & 0,60 & 19,67 & 3,07 &, 60 & 19,67 &, 44 &, 339 &, 363 \\
Juez30 & 3,25 & 0,44 & 13,57 & 3,21 &, 50 & 15,51 &,- 03 &, 399 &, 03 \\
\hline
\end{tabular}

Nota: N ítems: 28 . N válido según lista $=30$ jueces.

Fuente: Elaboración propia.

Cuadro 2. Estadísticos de los elementos y total-elemento de claridad y adecuación

\begin{tabular}{|c|c|c|c|c|c|c|c|c|c|c|}
\hline \multirow[b]{2}{*}{ JUECES } & \multicolumn{3}{|c|}{ CLARIDAD } & \multicolumn{3}{|c|}{ ADECUACIÓN } & \multicolumn{2}{|c|}{$\begin{array}{l}\text { CLARIDAD } \\
\end{array}$} & \multicolumn{2}{|c|}{$\begin{array}{l}\text { ADECUACIÓN } \\
\end{array}$} \\
\hline & Med. & $\begin{array}{l}\text { Des. } \\
\text { típica }\end{array}$ & CV & Med. & $\begin{array}{l}\text { Des. } \\
\text { típica }\end{array}$ & CV & $\begin{array}{l}\text { Correla. elemento- } \\
\text { total corregida }\end{array}$ & $\begin{array}{l}\text { Alfa de Cronbach si se } \\
\text { elimina el elemento }\end{array}$ & $\begin{array}{l}\text { Correla. elemento- } \\
\text { total corregida }\end{array}$ & $\begin{array}{c}\text { Alfa de Cronbach si se } \\
\text { elimina el elemento }\end{array}$ \\
\hline Juez 1 & 3,79 & 0,63 & 16,64 & 3,89 & 0,31 & 8,09 &,- 03 & ,380 & ,28 & ,232 \\
\hline Juez2 & 4,00 & 0,00 & 0,00 & 4,00 & 0,00 & 0,00 & ,OO & ,341 & ,OO & ,276 \\
\hline Juez3 & 3,71 & 0,60 & 16,15 & 3,43 & 0,88 & 25,63 &, 19 & ,303 & ,05 & ,294 \\
\hline Juez4 & 3,68 & 0,61 & 16,63 & 3,75 & 0,44 & 11,76 &, 16 & ,312 &,- 09 & ,313 \\
\hline Juez5 & 3,00 & 0,00 & 0,00 & 3,00 & 0,00 & 0,00 & ,OO &, 341 & ,OO &, 276 \\
\hline Juez6 & 3,93 & 0,38 & 9,62 & 3,39 & 0,69 & 20,20 & ,36 &, 278 &,$- O O$ & ,303 \\
\hline Juez7 & 3,96 & 0,19 & 4,77 & 3,96 & 0,19 & 4,77 &, 42 & ,306 &,- 06 & ,290 \\
\hline Juez8 & 2,36 & 0,62 & 26,37 & 2,57 & 0,69 & 26,84 &, 25 & ,280 &, 126 & ,249 \\
\hline Juez9 & 2,29 & 0,85 & 37,38 & 2,29 & 0,85 & 37,38 & ,OO & ,388 & 393 & ,083 \\
\hline Juez 10 & 4,00 & 0,00 & 0,00 & 4,00 & 0,00 & 0,00 & ,OO &, 341 & ,OO &, 276 \\
\hline Juez 11 & 3,93 & 0,26 & 6,68 & 3,68 & 0,48 & 12,93 & ,29 &, 310 &, 565 &, 113 \\
\hline Juez 12 & 3,93 & 0,38 & 9,62 & 3,93 & 0,26 & 6,67 &,- 17 & ,390 &, 142 & ,262 \\
\hline Juez 13 & 2,82 & 0,61 & 21,69 & 3,97 & 0,19 & 4,77 &, 34 & ,245 &,- 056 & ,290 \\
\hline Juez 14 & 4,00 & 0,00 & 0,00 & 4,00 & 0,00 & 0,00 & ,OO & 341 & ,OO &, 276 \\
\hline Juez 15 & 2,96 & 0,51 & 17,13 & 2,97 & 0,19 & 6,37 &, 13 &, 325 & , 019 &, 281 \\
\hline Juez 16 & 3,68 & 0,48 & 12,93 & 3,14 & 0,65 & 20,70 &, 44 &, 237 &, 157 & ,236 \\
\hline Juez 17 & $4, \mathrm{OO}$ & 0,00 & 0,00 & 4,00 & 0,00 & 0,00 & ,OO & 341 & ,OO &, 276 \\
\hline Juez 18 & 3,86 & 0,35 & 9,24 & 3,97 & 0,19 & 4,77 & 07 & 341 & ,095 & ,273 \\
\hline Juez 19 & 4,00 & 0,00 & 0,00 & 4,00 & 0,00 & 0,00 & ,00 &, 341 & ,OO &, 276 \\
\hline Juez20 & 3,89 & 0,57 & 14,56 & 3,86 & 0,36 & 9,24 &,- 03 & ,373 &,- 179 & ,324 \\
\hline Juez2 1 & 3,39 & 0,50 & 14,66 & 3,96 & 0,19 & 4,77 &,- 36 & 451 & ,019 & 281 \\
\hline Juez22 & 2,75 & 0,52 & 18,84 & 3,39 & 0,50 & 14,66 &,- 01 & ,366 & ,058 & ,275 \\
\hline
\end{tabular}




\begin{tabular}{ccccccccccc}
\hline Juez23 & 4,00 & 0,00 & 0,00 & 4,00 & 0,00 & 0,00 &, 00 &, 341 &, 00 &, 276 \\
Juez24 & 4,00 & 0,00 & 0,00 & 4,00 & 0,00 & 0,00 &, 00 &, 341 &, 00 \\
Juez25 & 3,68 & 0,48 & 12,93 & 2,75 & 0,65 & 23,47 &, 14 &, 322 &, 276 \\
Juez26 & 3,93 & 0,26 & 6,68 & 3,32 & 0,77 & 23,25 &, 07 &, 342 &, 348 \\
\hline
\end{tabular}

Nota: $\mathrm{N}$ ítems: $28 . \mathrm{N}$ válido según lista $=26$ jueces.

Fuente: Elaboración propia.

Cuadro 3. Estadísticos descriptivos. Escala de relevancia

\begin{tabular}{|c|c|c|c|c|c|c|c|c|c|c|c|}
\hline & \multicolumn{5}{|c|}{ TOTALES } & \multicolumn{3}{|c|}{ DEPURADOS } & \multicolumn{3}{|c|}{ DIFERENCIALES } \\
\hline & Mín. & Máx. & Media & Desv. Típica & CV & Media & Desv. Típica & CV & Media & Desv. Típica & $\mathrm{CV}$ \\
\hline It1Rel & 2 & 4 & 3,57 & 0,57 & 15,91 & 3,62 &, 590 & 16,298 & 3,44 &, 527 & 15,319 \\
\hline It2Rel & 1 & 4 & 3,47 & 0,82 & 23,60 & 3,52 &, 873 & 24,801 & 3,33 &, 707 & 21,231 \\
\hline It3Rel & 1 & 4 & 3,5 & 0,82 & 23,43 & 3,52 &, 873 & 24,801 & 3,44 & ,726 & 21,104 \\
\hline It4Rel & 1 & 4 & 3,4 & 0,93 & 27,41 & 3,33 & 1,017 & 30,54 & 3,56 &, 726 & 20,393 \\
\hline It5Rel & 2 & 4 & 3,5 & 0,68 & 19,49 & 3,48 &, 680 & 19,54 & 3,56 & ,726 & 20,393 \\
\hline It6Rel & 2 & 4 & 3,63 & 0,62 & 16,94 & 3,71 &, 561 & 15,121 & 3,44 & ,726 & 21,104 \\
\hline It7Rel & 2 & 4 & 3,37 & 0,72 & 21,31 & 3,38 & 740 & 21,893 & 3,33 & 707 & 21,231 \\
\hline It8Rel & 1 & 4 & 3,43 & 0,86 & 25,01 & 3,33 & ,966 & 29,009 & 3,67 &, 500 & 13,623 \\
\hline It9Rel & 1 & 4 & 3,4 & 0,72 & 21,29 & 3,43 & ,746 & 21,749 & 3,33 & ,707 & 21,231 \\
\hline It 10Rel & 3 & 4 & 3,7 & 0,47 & 12,59 & 3,76 & , 436 & 11,595 & 3,56 &, 527 & 14,803 \\
\hline It 11 Rel & 3 & 4 & 3,63 & 0,49 & 13,50 & 3,67 & , 483 & 13,16 & 3,56 &, 527 & 14,803 \\
\hline It 12Rel & 1 & 4 & 3,53 & 0,78 & 21,98 & 3,57 & ,746 & 20,896 & 3,44 &, 882 & 25,639 \\
\hline It13Rel & 1 & 4 & 3,5 & 0,78 & 22,20 & 3,52 & ,680 & 19,318 & 3,44 & 1,014 & 29,476 \\
\hline It 14Rel & 2 & 4 & 3,57 & 0,57 & 15,910 & 3,57 &, 507 & 14,201 & 3,56 &, 726 & 20,393 \\
\hline It 15Rel & 3 & 4 & 3,7 & 0,47 & 12,59 & 3,71 & , 463 & 12,479 & 3,67 &, 500 & 13,623 \\
\hline It16Rel & 1 & 4 & 3,47 & 0,82 & 23,60 & 3,48 & 873 & 25,086 & 3,44 & ,726 & 21,104 \\
\hline It 17 Rel & 1 & 4 & 3,33 & 0,88 & 26,55 & 3,29 & ,956 & 29,057 & 3,44 & ,726 & 21,104 \\
\hline It 18 Rel & 3 & 4 & 3,77 & 0,43 & 11,41 & 3,86 & ,359 & 9,3 & 3,56 &, 527 & 14,803 \\
\hline It 19Rel & 2 & 4 & 3,6 & 0,56 & 15,64 & 3,57 & ,598 & 16,75 & 3,67 &, 500 & 13,623 \\
\hline It2oRel & 1 & 4 & 3,43 & 0,82 & 23,82 & 3,38 & ,865 & 25,591 & 3,56 & ,726 & 20,393 \\
\hline It 21 Rel & 1 & 4 & 3,43 & 0,90 & 26,18 & 3,33 & 1,017 & 30,54 & 3,67 &, 500 & 13,623 \\
\hline It22Rel & 3 & 4 & 3,7 & 0,47 & 12,59 & 3,76 & ,436 & 11,595 & 3,56 &, 527 & 14,803 \\
\hline It23Rel & 2 & 4 & 3,6 & 0,62 & 17,25 & 3,76 &, 539 & 14,335 & 3,22 & 667 & 20,714 \\
\hline
\end{tabular}




\begin{tabular}{|c|c|c|c|c|c|c|c|c|c|c|c|}
\hline It24Rel & 2 & 4 & 3,57 & 0,63 & 17,53 & 3,57 & ,676 & 18,935 & 3,56 &, 527 & 14,803 \\
\hline It25Rel & 2 & 4 & 3,7 & 0,54 & 14,46 & 3,71 &, 561 & 15,121 & 3,67 &, 500 & 13,623 \\
\hline It26Rel & 2 & 4 & 3,6 & 0,56 & 15,64 & 3,76 & , 436 & 11,595 & 3,22 & ,667 & 20,714 \\
\hline It 27 Rel & 2 & 4 & 3,47 & 0,63 & 18,13 & 3,57 &, 598 & 16,75 & 3,22 & 667 & 20,714 \\
\hline It28Rel & 2 & 4 & 3,63 & 0,62 & 16,94 & 3,81 &, 402 & 10,551 & 3,22 & ,833 & 25,869 \\
\hline
\end{tabular}

Nota: válido según lista $=30$ jueces.

Fuente: Elaboración propia.

Cuadro 4. Estadísticos descriptivos. Escala de susceptibilidad de cambio

\begin{tabular}{|c|c|c|c|c|c|c|c|c|c|c|c|}
\hline & \multicolumn{5}{|c|}{ TOTALES } & \multicolumn{3}{|c|}{ DEPURADOS } & \multicolumn{3}{|c|}{ DIFERENCIALES } \\
\hline & Mín. & Máx. & Media & Desv. Típica & $\mathrm{CV}$ & Media & Desv. Típica & $\mathrm{CV}$ & Media & Desv. Típica & $\mathrm{CV}$ \\
\hline It1Sus & 2 & 4 & 3,67 & 0,55 & 14,90 & 3,70 &, 559 & 15,108 & 3,57 &, 535 & 14,985 \\
\hline It 2 Sus & 2 & 4 & 3,67 & 0,61 & 16,51 & 3,70 &, 559 & 15,108 & 3,57 & ,787 & 22,044 \\
\hline It3Sus & 2 & 4 & 3,57 & 0,73 & 20,39 & 3,57 & ,728 & 20,392 & 3,57 & ,787 & 22,044 \\
\hline It4Sus & 2 & 4 & 3,6 & 0,68 & 18,75 & 3,57 & ,728 & 20,392 & 3,71 & , 488 & 13,153 \\
\hline It5Sus & 2 & 4 & 3,67 & 0,66 & 18,011 & 3,61 & ,722 & 20 & 3,86 & ,378 & 9,792 \\
\hline It6Sus & 1 & 4 & 3,5 & 0,86 & 24,60 & 3,39 &, 941 & 27,758 & 3,86 & ,378 & 9,792 \\
\hline It7Sus & 1 & 4 & 3,5 & 0,78 & 22,20 & 3,48 & ,790 & 22,701 & 3,57 & ,787 & 22,044 \\
\hline It8Sus & 1 & 4 & 3,43 & 0,82 & 23,82 & 3,35 &, 885 & 26,417 & 3,71 & , 488 & 13,153 \\
\hline It9Sus & 1 & 4 & 3,53 & 0,68 & 19,29 & 3,48 & ,730 & 20,977 & 3,71 & , 488 & 13,153 \\
\hline It1oSus & 2 & 4 & 3,67 & 0,55 & 14,90 & 3,70 & ,470 & 12,702 & 3,57 & ,787 & 22,044 \\
\hline It 11 Sus & 2 & 4 & 3,67 & 0,55 & 14,90 & 3,65 & ,573 & 15,698 & 3,71 & , 488 & 13,153 \\
\hline It 12 Sus & 1 & 4 & 3,53 & 0,73 & 20,68 & 3,48 & ,790 & 22,701 & 3,71 & ,488 & 13,153 \\
\hline It 13Sus & 1 & 4 & 3,43 & 0,77 & 22,57 & 3,52 & ,593 & 16,846 & 3,14 & 1,215 & 38,694 \\
\hline It 14 Sus & 2 & 4 & 3,6 & 0,56 & 15,64 & 3,57 &, 590 & 16,526 & 3,71 & ,488 & 13,153 \\
\hline It 15 Sus & 2 & 4 & 3,63 & 0,56 & 15,32 & 3,61 & ,583 & 16,149 & 3,71 & ,488 & 13,153 \\
\hline It16Sus & 1 & 4 & 3,53 & 0,73 & 20,68 & 3,48 & ,790 & 22,701 & 3,71 & , 488 & 13,153 \\
\hline It 17 Sus & 1 & 4 & 3,33 & 0,84 & 25,35 & 3,22 & ,902 & 28,012 & 3,71 & , 488 & 13,153 \\
\hline It 18 Sus & 3 & 4 & 3,73 & 0,45 & 12,06 & 3,70 & , 470 & 12,702 & 3,86 & ,378 & 9,792 \\
\hline It19Sus & 3 & 4 & 3,7 & 0,47 & 12,59 & 3,70 & ,470 & 12,702 & 3,71 & 488 & 13,153 \\
\hline It2oSus & 2 & 4 & 3,63 & 0,62 & 16,94 & 3,61 & 656 & 18,171 & 3,71 & 488 & 13,153 \\
\hline It $21 \mathrm{Sus}$ & 1 & 4 & 3,6 & 0,68 & 18,75 & 3,57 & ,728 & 20,392 & 3,71 & , 488 & 13,153 \\
\hline It 22 Sus & 2 & 4 & 3,57 & 0,73 & 20,39 & 3,61 & ,656 & 18,171 & 3,43 & ,976 & 28,454 \\
\hline
\end{tabular}




\begin{tabular}{|c|c|c|c|c|c|c|c|c|c|c|c|}
\hline It23Sus & 2 & 4 & 3,47 & 0,73 & 21,04 & 3,48 & ,665 & 19,109 & 3,43 & ,976 & 28,454 \\
\hline It 24 Sus & 2 & 4 & 3,57 & 0,57 & 15,91 & 3,57 & ,590 & 16,526 & 3,57 & ,535 & 14,985 \\
\hline It25Sus & 2 & 4 & 3,73 & 0,52 & 13,97 & 3,65 &, 573 & 15,698 & 4,00 & ,000 & O \\
\hline It26Sus & 2 & 4 & 3,53 & 0,68 & 19,29 & 3,61 & 656 & 18,171 & 3,29 & 756 & 22,978 \\
\hline It $27 \mathrm{Sus}$ & 1 & 4 & 3,47 & 0,82 & 23,60 & 3,57 &, 843 & 23,613 & 3,14 &, 690 & 21,974 \\
\hline It 28 Sus & 2 & 4 & 3,67 & 0,61 & 16,51 & 3,61 & ,656 & 18,171 & 3,86 & ,378 & 9,792 \\
\hline
\end{tabular}

Nota: válido según lista $=26$ jueces.

Fuente: Elaboración propia.

Cuadro 5. Estadísticos descriptivos. Escala de susceptibilidad de claridad

\begin{tabular}{|c|c|c|c|c|c|c|c|c|c|c|c|}
\hline & \multicolumn{5}{|c|}{ Totales } & \multicolumn{3}{|c|}{ DEPURADOS } & \multicolumn{3}{|c|}{ DIFERENCIALES } \\
\hline & Mín. & Máx. & Media & Desv. típ. & $\mathrm{CV}$ & Media & Desv.Típica & $\mathrm{CV}$ & Media & Desv. Típica & $\mathrm{CV}$ \\
\hline It $1 \mathrm{Cla}$ & 2 & 4 & 3,65 & 0,56 & 15,40 & 3,72 &, 575 & 15,456 & 3,50 &, 535 & 15,285 \\
\hline It $2 \mathrm{Cla}$ & 2 & 4 & 3,69 & 0,55 & 14,88 & 3,83 & ,383 & 10 & 3,38 &, 744 & 22,011 \\
\hline It3Cla & 2 & 4 & 3,58 & 0,70 & 19,64 & 3,61 & 608 & 16,842 & 3,50 & ,926 & 26,457 \\
\hline It $4 \mathrm{Cla}$ & 2 & 4 & 3,65 & 0,69 & 18,88 & 3,78 & 548 & 14,497 & 3,38 & 916 & 27,1 \\
\hline It5Cla & 2 & 4 & 3,69 & 0,68 & 18,40 & 3,78 &, 548 & 14,497 & 3,50 & ,926 & 26,457 \\
\hline It6Cla & 1 & 4 & 3,5 & 0,91 & 25,89 & 3,56 & 856 & 24,044 & 3,38 & 1,061 & 31,39 \\
\hline It7Cla & 1 & 4 & 3,58 & 0,76 & 21,17 & 3,67 & ,767 & 20,899 & 3,38 &, 744 & 22,011 \\
\hline It $8 \mathrm{Cla}$ & 1 & 4 & 3,46 & 0,86 & 24,83 & 3,50 & ,786 & 22,457 & 3,38 & 1,061 & 31,39 \\
\hline It9Cla & 1 & 4 & 3,58 & 0,70 & 19,64 & 3,67 & 485 & 13,215 & 3,38 & 1,061 & 31,39 \\
\hline It $10 \mathrm{Cla}$ & 2 & 4 & 3,69 & 0,55 & 14,88 & 3,78 & 428 & 11,322 & 3,50 &, 756 & 21,6 \\
\hline It $11 \mathrm{Cla}$ & 2 & 4 & 3,69 & 0,55 & 14,88 & 3,72 &, 575 & 15,456 & 3,63 &, 518 & 14,269 \\
\hline It $12 \mathrm{Cla}$ & 1 & 4 & 3,58 & 0,76 & 21,17 & 3,67 & 594 & 16,185 & 3,38 & 1,061 & 31,39 \\
\hline It $13 \mathrm{Cla}$ & 1 & 4 & 3,5 & 0,76 & 21,77 & 3,67 & 594 & 16,185 & 3,13 & ,991 & 31,661 \\
\hline It $14 \mathrm{Cla}$ & 2 & 4 & 3,65 & 0,56 & 15,40 & 3,67 &, 594 & 16,185 & 3,63 &, 518 & 14,269 \\
\hline It $15 \mathrm{Cla}$ & 2 & 4 & 3,65 & 0,56 & 15,40 & 3,67 & 594 & 16,185 & 3,63 & 518 & 14,269 \\
\hline It $16 \mathrm{Cla}$ & 1 & 4 & 3,58 & 0,76 & 21,17 & 3,67 &, 594 & 16,185 & 3,38 & 1,061 & 31,39 \\
\hline It $17 \mathrm{Cla}$ & 1 & 4 & 3,35 & 0,85 & 25,25 & 3,44 & ,705 & 20,494 & 3,13 & 1,126 & 35,974 \\
\hline It $18 \mathrm{Cla}$ & 3 & 4 & 3,77 & 0,43 & 11,41 & 3,78 & 428 & 11,322 & 3,75 & ,463 & 12,346 \\
\hline It $19 \mathrm{Cla}$ & 3 & 4 & 3,73 & 0,45 & 12,12 & 3,78 & 428 & 11,322 & 3,63 &, 518 & 14,269 \\
\hline It $20 \mathrm{Cla}$ & 2 & 4 & 3,65 & 0,63 & 17,23 & 3,78 & 428 & 11,322 & 3,38 & ,916 & 27,1 \\
\hline It $21 \mathrm{Cla}$ & 1 & 4 & 3,65 & 0,69 & 18,88 & 3,78 & 428 & 11,322 & 3,38 & 1,061 & 31,39 \\
\hline
\end{tabular}




\begin{tabular}{|c|c|c|c|c|c|c|c|c|c|c|c|}
\hline It22Cla & 2 & 4 & 3,5 & 0,76 & 21,77 & 3,39 &, 850 & 25,073 & 3,75 & ,463 & 12,346 \\
\hline It $23 \mathrm{Cla}$ & 2 & 4 & 3,5 & 0,76 & 21,77 & 3,39 &, 850 & 25,073 & 3,75 & , 463 & 12,346 \\
\hline It $24 \mathrm{Cla}$ & 2 & 4 & 3,58 & 0,58 & 16,15 & 3,56 &, 511 & 14,353 & 3,63 &, 744 & 20,495 \\
\hline It25Cla & 2 & 4 & 3,73 & 0,53 & 14,29 & 3,78 & 428 & 11,322 & 3,63 & 744 & 20,495 \\
\hline It $26 \mathrm{Cla}$ & 2 & 4 & 3,5 & 0,71 & 20,20 & 3,50 &, 707 & 20,2 & 3,50 &, 756 & 21,6 \\
\hline It $27 \mathrm{Cla}$ & 1 & 4 & 3,42 & 0,86 & 25,06 & 3,44 & ,856 & 24,883 & 3,38 & ,916 & 27,1 \\
\hline It $28 \mathrm{Cla}$ & 2 & 4 & 3,62 & 0,64 & 17,60 & 3,67 &, 594 & 16,185 & 3,50 & ,756 & 21,6 \\
\hline
\end{tabular}

Nota: válido según lista =26 jueces.

Fuente: Elaboración propia.

Cuadro 6. Estadísticos descriptivos. Escala de susceptibilidad de adecuación

\begin{tabular}{|c|c|c|c|c|c|c|c|c|c|c|c|}
\hline & \multicolumn{5}{|c|}{ Totales } & \multicolumn{3}{|c|}{ DEPURADOS } & \multicolumn{3}{|c|}{ DIFERENCIALES } \\
\hline & Mín. & Máx. & Media & Desv. Típica & $\mathrm{CV}$ & Media & Desv. Típica & $\mathrm{CV}$ & Media & Desv. Típica & $\mathrm{CV}$ \\
\hline It1Ad & 2 & 4 & 3,54 & 0,58 & 16,44 & 3,53 &, 624 & 17,677 & 3,56 &, 527 & 14,803 \\
\hline It $2 \mathrm{Ad}$ & 2 & 4 & 3,58 & 0,64 & 17,96 & 3,65 & ,606 & 16,602 & 3,44 & ,726 & 21,104 \\
\hline It3Ad & 1 & 4 & 3,5 & 0,81 & 23,20 & 3,41 &, 870 & 25,513 & 3,67 & ,707 & 19,264 \\
\hline It $4 \mathrm{Ad}$ & 1 & 4 & 3,5 & 0,86 & 24,57 & 3,59 &, 870 & 24,233 & 3,33 & ,866 & 26,006 \\
\hline It5Ad & 2 & 4 & 3,58 & 0,70 & 19,64 & 3,71 & ,588 & 15,849 & 3,33 & ,866 & 26,006 \\
\hline It6Ad & 2 & 4 & 3,69 & 0,62 & 16,75 & 3,71 & ,588 & 15,849 & 3,67 & ,707 & 19,264 \\
\hline It7Ad & 2 & 4 & 3,42 & 0,70 & 20,56 & 3,59 & ,618 & 17,214 & 3,11 & ,782 & 25,144 \\
\hline It $8 \mathrm{Ad}$ & 1 & 4 & 3,46 & 0,91 & 26,16 & 3,53 & 1,007 & 28,526 & 3,33 & ,707 & 21,231 \\
\hline It9Ad & 1 & 4 & 3,46 & 0,76 & 21,99 & 3,59 &, 795 & 22,144 & 3,22 & 667 & 20,714 \\
\hline It 10Ad & 3 & 4 & 3,73 & 0,45 & 12,12 & 3,76 & , 437 & 11,622 & 3,67 &, 500 & 13,623 \\
\hline t11 Ad & 3 & 4 & 3,69 & 0,47 & 12,76 & 3,76 & 437 & 11,622 & 3,56 &, 527 & 14,803 \\
\hline It $12 \mathrm{Ad}$ & 1 & 4 & 3,54 & 0,81 & 22,91 & 3,71 & ,772 & 20,808 & 3,22 & ,833 & 25,869 \\
\hline It $13 \mathrm{Ad}$ & 1 & 4 & 3,54 & 0,76 & 21,50 & 3,76 & ,562 & 14,946 & 3,11 & ,928 & 29,839 \\
\hline It $14 \mathrm{Ad}$ & 2 & 4 & 3,65 & 0,56 & 15,40 & 3,82 & 393 & 10,287 & 3,33 & ,707 & 21,231 \\
\hline It $15 \mathrm{Ad}$ & 3 & 4 & 3,73 & 0,45 & 12,12 & 3,82 & 393, & 10,287 & 3,56 &, 527 & 14,803 \\
\hline It16Ad & 1 & 4 & 3,5 & 0,86 & 24,57 & 3,53 & ,943 & 26,713 & 3,44 & ,726 & 21,104 \\
\hline It $17 \mathrm{Ad}$ & 1 & 4 & 3,46 & 0,81 & 23,44 & 3,53 & ,874 & 24,759 & 3,33 & ,707 & 21,231 \\
\hline It $18 \mathrm{Ad}$ & 3 & 4 & 3,77 & 0,43 & 11,41 & 3,82 & ,393 & 10,287 & 3,67 &, 500 & 13,623 \\
\hline It19Ad & 2 & 4 & 3,65 & 0,56 & 15,40 & 3,82 & ,393 & 10,287 & 3,33 & ,707 & 21,231 \\
\hline It2oAd & 2 & 4 & 3,58 & 0,70 & 19,64 & 3,76 &, 562 & 14,946 & 3,22 & ,833 & 25,869 \\
\hline
\end{tabular}




\begin{tabular}{|c|c|c|c|c|c|c|c|c|c|c|c|}
\hline It $21 \mathrm{Ad}$ & 1 & 4 & 3,54 & 0,81 & 22,91 & 3,65 & ,862 & 23,616 & 3,33 & ,707 & 21,231 \\
\hline It $22 \mathrm{Ad}$ & 3 & 4 & 3,69 & 0,47 & 12,76 & 3,71 & 470 & 12,668 & 3,67 &, 500 & 13,623 \\
\hline It $23 \mathrm{Ad}$ & 2 & 4 & 3,58 & 0,64 & 17,96 & 3,65 & ,606 & 16,602 & 3,44 & ,726 & 21,104 \\
\hline It $24 \mathrm{Ad}$ & 2 & 4 & 3,69 & 0,55 & 14,88 & 3,71 &, 588 & 15,849 & 3,67 &, 500 & 13,623 \\
\hline It $25 \mathrm{Ad}$ & 2 & 4 & 3,73 & 0,53 & 14,29 & 3,76 &, 562 & 14,946 & 3,67 &, 500 & 13,623 \\
\hline It $26 \mathrm{Ad}$ & 2 & 4 & 3,54 & 0,58 & 16,44 & 3,59 &, 507 & 14,122 & 3,44 & ,726 & 21,104 \\
\hline It $27 \mathrm{Ad}$ & 2 & 4 & 3,46 & 0,65 & 18,70 & 3,47 &, 624 & 17,982 & 3,44 &, 726 & 21,104 \\
\hline It $28 \mathrm{Ad}$ & 2 & 4 & 3,58 & 0,64 & 17,96 & 3,65 & ,606 & 16,602 & 3,44 & ,726 & 21,104 \\
\hline
\end{tabular}

Nota: válido según lista $=30$ jueces

Fuente: Elaboración propia. 


\section{Breve CV de los autores}

\section{Anna Jordina Llorens}

Magister en Política, Gestión y Dirección de los Centros Educativos por la Universitat de València (con Premio Extraordinario) y graduada en Magisterio Primaria con Mención en Educación Física en la Universitat Jaume I de Castelló de la Plana. Actualmente colabora como investigadora del Departamento de Métodos de Investigación y Diagnóstico en Educación (MIDE) en la Facultad de Filosofía y Ciencias de la Educación, Universitat de València, y es miembro del Grupo de Evaluación y Medición GEM-EduCo (www.uv.es/gem/gemeduco). Actualmente desarrolla su investigación de doctorado en torno al tema de evaluación de la dimensión de Justicia Social Percibida por el alumnado de primaria y secundaria, en el marco de la evaluación de Sistemas Educativos para la Cohesión Social. ORCID ID: https://orcid.org/0000-0002-5402-5129. Email: anjorllo@alumni.uv.es

\section{Jesús Miguel Jornet Meliá}

Licenciado y doctor en Filosofía y Ciencias de la Educación por la Universidad de Valencia, España. Catedrático de la Universitat de València, desde 2006. Coordinador del Grupo GemEduCo y miembro del Grupo de Innovación Docente InnovaMIDE. Ha sido miembro Consejo Técnico del INEE, y actualmente pertenece a diversas asociaciones nacionales y europeas de investigación y evaluación educativas (SEP, AIDIPE, EERA, AEOP, RIIED) y es asesor de diversas revistas de investigación y evaluación educativas. Líneas de investigación: de medición (construcción y validación de instrumentos) y evaluación (diseño y desarrollo de sistemas de evaluación) en educación. Para más información http://www.uv.es/gem/gemeduco/. ORCID ID: https://orcid.org/ooooo001-6905-497X. Email: jornet@uv.es

\section{Purificación Sánchez Delgado}

Doctora en Ciencias de la Educación. Profesora Contratada Doctora de la Universitat de València del área de Medición y Evaluación Educativa. Ha impartido docencia en el área de la educación y sobre evaluación e intervención y metodología de evaluación de programas en el área de la logopedia. Miembro del Grupo de Evaluación y Medición (GEM) (www.uv.es/gem). En el ámbito de la medición educativa trabaja en el desarrollo y validación de pruebas para medir diferentes tipos de variables educativas vinculadas al área del lenguaje. En el ámbito de la evaluación, trabaja tanto en evaluación de sistemas educativos como evaluación de aprendizajes y competencias. ORCID ID: https://orcid.org/o000-0003-4443-8904.Email: purificacion.sanchez@uv.es 